Publ. RIMS, Kyoto Univ.

15 (1979), 159-199

\title{
A Mathematical Model of Spherical Nucleus in the Undercooling State of Metal
}

By

Tatsuo NOGI*

\section{$\S 1$. Introduction and Formulation**}

It is well known that when a pure liquid is cooled beyond its melting point $T_{E}$, the solid phase may form; alternatively if nucleation is suppressed, the liquid may continue to cool (that is, to undercool or supercool).

The free energy change on forming solid from liquid at the equilibrium transformation temperature $T_{E}$ is equal to zero:

$$
\Delta G=\Delta H-T_{E} d S=0
$$

where $\Delta G, \Delta H$ and $J S$ are molar changes in free energy, enthalpy and entropy, respectively. At any temperature $T$ different from $T_{E}, J G$ is not equal to zero:

$$
\lrcorner G=\lrcorner H-T \cdot S \neq 0 .
$$

Neglecting the small temperature-dependence of $\Delta H$ and $\Delta S$ and combining the above two equations we have

$$
\Delta G=\frac{\Delta H \Delta T}{T_{E}}
$$

where $\Delta T$ is the undercooling $\left(=T_{E}-T\right)$, whose value is negative for solidification.

An important influence on the melting point of a pure material is given by the surface curvature. The surface curvature can be considered to introduce excess pressure only in the solid phase. The excess pressure

Communicated by K. Itô, December 9, 1976.

* Department of Applied Mathematics and Physics, Faculty of Engineering, Kyoto University.

** About physical base we rely on [1]. 
is given as follows:

$$
\Delta p=2 \sigma \kappa
$$

where $\sigma$ is the surface free energy per unit area and $\kappa$ is the mean surface curvature. If the solid cannot be compressed, $\Delta p=\Delta G$ holds. Thus

$$
\frac{\Delta H \Delta T}{T_{E}}=2 \sigma \kappa
$$

or

$$
\frac{1}{\kappa}=\frac{2 \sigma T_{E}}{\Delta H \Delta T}=\frac{2 \sigma T_{E}}{L\left(T_{E}-T_{I}\right)}
$$

where $L$ is the latent heat and $T_{I}$ is the surface temperature.

A crystal smaller than a critical size is called "embryo", which grows to nucleus with the critical size. We are concerned about growth of the nucleus. For simplicity we consider a spherical nucleus, whose size is determined just by its radius. The critical radius is given by the formula

$$
r^{*}=\frac{2 \sigma T_{E}}{L\left(T_{E}-T_{A}\right)}
$$

where $T_{A}$ is the initial liquid temperature of undercool state.

From the above discussion we can describe a system of equations which determines the radius $Y(t)$ and the temperature distribution $T(r, t)$ as follows:

$$
\begin{cases}\rho c \frac{\partial T}{\partial t}=k \Delta T & (0<r<Y(t), t>0) \\ \frac{L\left(T_{E}-T_{T}\right)}{2 \sigma T_{E}}=\frac{1}{Y(t)} & (t>0) \\ L \rho \dot{Y}(t)=-k \frac{\partial T}{\partial r}(Y(t), t) & (t>0) \\ Y(0)=\frac{2 \sigma T_{E}}{L\left(T_{E}-T_{A}\right)}, & (0 \leq r \leq Y(0)) \\ T(r, 0)=T_{0}(r) & \end{cases}
$$

where $T_{I}=T(Y(t), t)$, and $k$ is thermal conductivity, $\rho$ density, $c$ specific heat of the solid metal, and $L$ latent heat, which are assumed to be constant. Putting 


$$
\begin{aligned}
& \frac{T-T_{A}}{T_{E}}=u, \frac{k T_{E}}{L \rho}=\alpha, \frac{2 \sigma}{L}=m, \\
& \frac{2 \sigma T_{E}}{L\left(T_{E}-T_{A}\right)}=b, \quad Y(t)=y(t) \text { and } T_{0}(r)=f(r),
\end{aligned}
$$

the above equations take the following normalized form:

$$
(\mathrm{P})\left\{\begin{array}{lll}
(\mathrm{P}-1) & \frac{\partial u}{\partial t}=\Delta u & (0<r<y(t), t>0), \\
(\mathrm{P}-2) & u(y(t), t)=\frac{m}{b}-\frac{m}{y(t)} & (t>0) \\
(\mathrm{P}-3) & \dot{y}(t)=-\alpha \frac{\partial u}{\partial r}(y(t), t) & (t>0) \\
(\mathrm{P}-4) & y(0)=b, & \\
(\mathrm{P}-5) & u(r, 0)=f(r) & (0 \leq r \leq b) .
\end{array}\right.
$$

Here we shall refer to the series of A. Friedman's works being entitled "Free boundary problems for parabolic equations", Part I [2], Part II [3] and Part III [4]. In Part I he considered the problem of melting of solids. In Part II he considered the development of one liquid drop surrounded by totally supersaturated vapour of its own substance. Denoting by $u(r, t)$ the normalized vapour density at each point $(r, t)$ and by $y(t)$ the normalized radius of the drop, we get the following equations of $u(r, t)$ and $v(t)$ :

$$
\begin{cases}\frac{\partial u}{\partial t}=\Delta u & (y(t)<r<\infty, t>0) \\ u(y(t), t)=1 & (t>0) \\ \dot{v}(t)=\alpha \frac{\partial u}{\partial r}(y(t), t) & (t>0) \\ v(0)=b>0, & \\ u(r, 0)=f(r) & (r>b) .\end{cases}
$$

In Part III he considered the behaviour of one gas bubble in liquid in which some part of the same gas is dissolved. Denoting by $u(r, t)$ the normalized density of the dissolved gas in the liquid and by $y(t)$ the normalized radius of the bubble, we get the following equations of $u(r, t)$ 
and $y(t)$ :

$$
\begin{cases}\frac{\partial u}{\partial t}=\Delta u & (y(t)<r<\infty, t>0) \\ u(y(t), t)=\frac{m}{y(t)}-\frac{m}{b} & (t>0) \\ \left(1+\frac{m}{y(t)}\right) \dot{y}(t)=\alpha \frac{\partial u}{\partial r}(y(t), t) & (t>0) \\ y(0)=b>0, & (r>b) .\end{cases}
$$

Note that the case of $m=0$ and $n=0$ reduces to that of part II by putting $1-u=v$. He could prove unique existence of the solution only for small $\alpha$.

Our problem is analogous to Friedman's problems, especially to that of Part III. Thus it may be considered as that of Part IV. Indeed we can prove unique existence of the solution by his method. Here we will state the outline. By putting

$$
r=x, r u(r, t)=w(x, t) \text { and } r f(r)=\phi(x),
$$

our system (P) takes the form

$$
\left(\mathrm{P}^{\prime}\right) \begin{cases}\frac{\partial w}{\partial t}=\frac{\partial^{2} w}{\partial x^{2}} & (0<x<y(t), t>0), \\ w(y(t), t)=\frac{m}{b}(y(t)-b) & (t>0), \\ y(0, t)=0 & (t>0), \\ y(t) \dot{y}(t)=-\alpha \frac{\partial w}{\partial x}(y(t), t)+\alpha\left(\frac{m}{b}-\frac{m}{y(t)}\right) & (t>0), \\ w(x, 0)=\phi(x) & (0<x<b) .\end{cases}
$$

By introducing the Green's functions of the heat equation

$$
\begin{aligned}
g(x, t ; \xi, \tau) & =\frac{1}{2 \sqrt{\pi(t-\tau)}} \exp \left(-\frac{(x-\xi)^{2}}{4(t-\tau)}\right)-\frac{1}{2 \sqrt{\pi(t-\tau)}} \exp \left(-\frac{(x+\xi)^{2}}{4(t-\tau)}\right) \\
G(x, t ; \xi, \tau) & =\frac{1}{2 \sqrt{\pi(t-\tau)}} \exp \left(-\frac{(x-\xi)^{2}}{4(t-\tau)}\right)+\frac{1}{2 \sqrt{\pi(t-\tau)}} \exp \left(-\frac{(x+\xi)^{2}}{4(t-\tau)}\right)
\end{aligned}
$$


and making use of the standard technique, we get the nonlinear integral equation of Volterra type for $v(t) \equiv u_{x}\left(\jmath^{\prime}(t)-0, t\right)$ equivalent to the last system:

$$
\left(\mathrm{P}^{\prime \prime}\right)\left\{\begin{aligned}
& v(t)=-2 \int_{0}^{b} G_{\xi}(y(t), t ; \xi, 0) \phi(\xi) d \xi \\
&+\frac{2 m}{b} \int_{0}^{t} \dot{y}(\tau) G(y(t), t ; y(\tau), \tau) d \tau \\
&-2 \int_{0}^{t} G_{\xi}(y(t), t ; y(\tau), \tau) v(\tau) d \tau \\
& y(t) \dot{y}(t)+\alpha\left(\frac{m}{y}-\frac{m}{b}\right)+\alpha v(t)=0 .
\end{aligned}\right.
$$

If the system $\left(\mathrm{P}^{\prime \prime}\right)$ for $v(t)$ and $y(t)$ is solved, then $w(x, t)$ is given by the formula

$$
\begin{aligned}
w(x, t)= & \int_{0}^{b} g(x, t ; \xi, 0) \phi(\xi) d \xi \\
& +\frac{m}{b} \int_{0}^{t} g(x, t ; y(\tau), \tau)(y(\tau)-b) \dot{y}(\tau) d \tau \\
& +\int_{0}^{t}\{g(x, t ; y(\tau), \tau) v(\tau) \\
& \left.-\frac{m}{b} u(y(\tau), \tau) \frac{\partial g}{\partial \xi}(x, t ; y(\tau), \tau)\right\} d \tau .
\end{aligned}
$$

The system $\left(\mathrm{P}^{\prime \prime}\right)$ can be solved by Picard's iteration procedure for small $t$ and small $\alpha$. By using the monotonicity of $y(t)$ (that itself is to be made sure of) the solution can be continued for all $t$.

In the above method, however, we must impose a severe restriction on smallness of $\alpha$. Therefore we will take another way in the following, which is analogous to that Cannon, Hill and Primicerio took for the simplest Stefan's problem ([5], [6] and [7]).

We consider the problem (P) in the classical sense. Its classical solution is defined as follows:

Definition. A pair of functions $(y, u)$ is called a solution of the system $(\mathrm{P})$ in $0 \leq t \leq T$, if the following conditions are satisfied:

i) $y(t) \in C(0 \leq t \leq T), \quad \dot{y}(t) \in C(0<t \leq T)$, 
ii) $u(r, t), \frac{\partial u}{\partial r}(r, t) \in C(0 \leq r \leq y(t), 0<t \leq T)$,

$$
\Delta u \in C(0 \leq r \leq y(t), 0<t \leq T),
$$

iii) $(y, u)$ satisfies $(\mathrm{P}-1) \sim(\mathrm{P}-5){ }^{*}$

In $\S 2$ we will give some lemmas necessary for later purposes, and in $\S 3$ uniqueness and existence theorems. In $\S 5$ we will propose a difference scheme for solving our problem numerically and give another proof of the existence theorem as a by-product. In $\S 6$ we will give an iteration procedure for solving our difference scheme and prove its convergence, and in $\S 7$ show some numerical examples by our computation.

\section{§ 2. Some Lemmas}

We will give some a priori properties of the solution of the problem (P). First we assume that $f(r)$ is smooth except for at most finite number of finite jumps at whose points $f^{\prime}(r-0)$ and $f^{\prime}(r+0)$ exist, and that

$$
\begin{array}{ll}
f(r) \geq 0, \quad f(r) \neq 0 & (0 \leq r \leq b), \\
f^{\prime}(r-0)+f^{\prime}(r+0) \leq 0 & (0 \leq r \leq b) .
\end{array}
$$

Lemma 1. If $\dot{y}(t) \geq 0$ for an interval $0 \leq t \leq t_{0}$, then

$$
\frac{\partial u}{\partial r}(r, t) \leq 0 \text { for } 0 \leq r \leq y(t), 0<t \leq t_{0} .
$$

Proof. Put $\frac{\partial u}{\partial r} \equiv \approx . \approx$ satisfies the system of equations

$$
\begin{cases}\Delta z-\frac{2}{r^{2}} z-\frac{\partial z}{\partial t}=0 & \left(0<r<y(t), 0<t \leq t_{0}\right), \\ z(y(t), t)=-\frac{1}{\alpha} \dot{y}(t) \leq 0 & \left(0<t \leq t_{0}\right),\end{cases}
$$

* At each discontinuous point $r_{0}$ of $f(r)$, we demand only that

$$
0 \leq \lim _{(r, t) \rightarrow\left(\overline{r_{0}}, 0\right)} u(r, t) \leq \varlimsup_{(r, t) \rightarrow\left(r_{0}, 0\right)}^{-} u(r, t) \leq f\left(r_{0}\right) .
$$




$$
\mid \begin{array}{lr}
z(r, 0)=\frac{1}{2}\left(f^{\prime}(r+0)+f^{\prime}(r-0)\right) \leq 0 & (0<r<b), \\
z(0, t)=0 & \left(0<t \leq t_{0}\right) .
\end{array}
$$

Suppose that there exists an interior point $(\bar{r}, \bar{t})(0<\bar{r}<y(\bar{t}), \bar{t}>0)$ such that

$$
\max _{\substack{0 \leq r \leq y(t) \\ 0 \leq t \leq t_{0}}} z(r, t)=z(\bar{r}, \bar{t})=M>0, \quad z(r, t)<M(t<\bar{t})
$$

Then at $(\bar{r}, \bar{t}), z_{r}=0, z_{r r} \leq 0$ and $z_{t} \geq 0$ hold, but this implies that $\Delta z$ $-\frac{2}{r^{2}} z-z_{l}<0$ at $(\bar{r}, \bar{t})$, which is a contradiction. Therefore it should hold that $z(r, t)=\frac{\partial u}{\partial r}(r, t) \leq 0$ for $0 \leq r \leq y(t), 0<t \leq t_{0}$. $\quad$ Q.E.D.

Lemma 2. Under the same assumption of $f(r)$ as above, $y(t)$ is strictly' monotone increasing for $t \geq 0$. And hence

$$
\frac{\partial u}{\partial r}(r, t) \leq 0 \quad \text { for } 0 \leq r \leq y(t), \quad t>0
$$

Proof. Assume that $\dot{y}(t)<0$ in an interval $0<t \leq t_{0}$. We consider a function $U$ defined by

$$
U=u-\left(\frac{m}{b}-\frac{m}{y(t)}\right)
$$

which satisfies the system of equations

$$
\left\{\begin{array}{l}
\Delta U-U_{t}=\frac{m \dot{y}(t)}{y(t)^{2}}(<0) \quad\left(0<r<y(t), 0<t \leq t_{0}\right) \\
U(y(t), t)=0 \\
U(r, 0)=f(r) \quad(\geq 0) .
\end{array}\right.
$$

By the well-known maximum principle (or by a similar way to that in the proof of Lemma 1), we have $U \geq 0(0 \leq r \leq y(t))$, and hence $\frac{\partial u}{\partial r}(y(t), t)$ $=\frac{\partial U}{\partial r}(y(t), t) \leq 0 . \quad$ This means $\dot{y}(t) \geq 0 \quad\left(0<t \leq t_{0}\right)$ by $\quad(\mathrm{P}-3)$, which is, however, a contradiction to our assumption $\dot{y}(t)<0$. Thus we should have $\dot{y}(t) \geq 0$ for some interval $0<t \leq t_{0}$.

Next we assume that there exists an interval $t_{0}<t \leq t_{1} \quad\left(t_{0}>0\right)$ for which $\dot{y}(t)<0$ and for $0<t \leq t_{0}, \dot{y}(t) \geq 0$. From Lemma 1 we have 


$$
U\left(r, t_{0}\right)=u\left(r, t_{0}\right)-\left(\frac{m}{b}-\frac{m}{y\left(t_{0}\right)}\right) \geq 0
$$

Hence we again arrive at $U \geq 0\left(0 \leq r \leq y(t), t_{0} \leq t \leq t_{1}\right)$ which contradicts the assumption $\dot{y}(t)<0 \quad\left(t_{0}<t \leq t_{1}\right)$. Thus we should have $\dot{y}(t) \geq 0$ for all $t \geq 0$.

Now we will show the strict monotonicity of $y(t)$. If the contrary were to happen, there would be $t^{\prime}$ and $t^{\prime \prime}$ such that $v(t) \equiv y\left(t^{\prime}\right)\left(t^{\prime} \leq t\right.$ $\left.\leq t^{\prime \prime}\right)$ and hence $\dot{y}(t)=0\left(t^{\prime} \leq t \leq t^{\prime \prime}\right)$. On the other hand we get $U>0$ from (2.1) by the maximum principle and hence $\frac{\partial u}{\partial r}(y(t), t)=\frac{\partial U}{\partial r}(y(t), t)$ $<0 \quad\left(t^{\prime} \leq t \leq t^{\prime \prime}\right)$ by Friedman's lemma [8], which is a contradiction to $\dot{y}(t)=0 \quad\left(t^{\prime} \leq t \leq t^{\prime \prime}\right)$.

The last statement of Lemma 2 follows directly from Lemma 1 . Thus the proof is completed.

Lemma 3. Let $\left(y_{i}, u_{i}\right) \quad(i=1,2)$ be the solutions of $(\mathrm{P})$ corresponding to two pairs of data $\left(b_{i}, f_{i}\right) \quad(i=1,2)$ satisfying $(2.1)$, respectively. If $b_{1}<b_{2}$ and $f_{1} \leq f_{2}\left(0 \leq r \leq b_{2}\right)$ (where $f_{1}$ is assumed to be extended and to be equal to zero for $\left.b_{1} \leq r \leq b_{2}\right)$, then

$$
y_{1}(t)<y_{2}(t) \quad(t>0) .
$$

Proof. If the statement is not true, there is a $t_{0}(>0)$ such that $y_{1}\left(t_{0}\right)=y_{2}\left(t_{0}\right), \dot{y}_{1}\left(t_{0}\right) \geq \dot{y}_{2}\left(t_{0}\right)$ and $y_{1}(t)<y_{2}(t) \quad\left(0 \leq t \leq t_{0}\right)$. In $0<t<t_{0}$, we have by Lemma 2

$$
u_{2}\left(y_{1}(t), t\right) \geq \frac{m}{b}-\frac{m}{y_{2}(t)}>\frac{m}{b}-\frac{m}{y_{1}(t)}=u_{1}\left(y_{1}(t), t\right)
$$

and hence by the maximum principle

$$
u_{2}(r, t)-u_{1}(r, t)>0 \quad\left(0<r<y_{1}(t), \quad 0<t<t_{0}\right) .
$$

Since $u_{2}\left(y_{1}\left(t_{0}\right), t_{0}\right)-u_{1}\left(y_{1}\left(t_{0}\right), t_{0}\right)=0$, it follows by Friedman's lemma that

$$
\frac{\partial u_{2}}{\partial r}\left(y_{2}\left(t_{0}\right), t_{0}\right)-\frac{\partial u_{1}}{\partial r}\left(y_{1}\left(t_{0}\right), t_{0}\right)<0, \quad \text { i.e. } \dot{y}_{2}\left(t_{0}\right)>\dot{y}_{1}\left(t_{0}\right)
$$

which is a contradiction. Thus we have completed the proof.

Now we will give a fundamental formula useful for later purposes. 
By multiplying $(\mathrm{P}-1)$ by $r^{2}$ and integrating the result over the region $(0<r<y(\tau), 0<\tau<t)$ we have

$$
\begin{gathered}
\int_{0}^{y(t)} u(r, t) r^{2} d r-\int_{0}^{b} u(r, 0) r^{2} d r-\int_{0}^{t} u(y(\tau), \tau) y^{2}(\tau) \dot{y}(\tau) d \tau \\
=\int_{0}^{t} y^{2}(\tau) \frac{\partial u}{\partial r}(y(\tau), \tau) d \tau
\end{gathered}
$$

which can be written by using the conditions $(\mathrm{P}-2) \sim(\mathrm{P}-5)$ as

$$
\begin{gathered}
\int_{0}^{y(t)} u(r, t) r^{2} d r-\int_{0}^{b} f(r) r^{2} d r-\int_{0}^{t}\left(\frac{m}{b}-\frac{m}{y(\tau)}\right) y^{2}(\tau) \dot{y}(\tau) d \tau \\
=-\frac{1}{\alpha} \int_{0}^{t} y^{2}(\tau) \dot{y}(\tau) d \tau .
\end{gathered}
$$

From this and by integration we get the formula

$$
\begin{aligned}
\frac{1}{3}\left(\frac{1}{\alpha}-\frac{m}{b}\right)\left(y^{3}(t)-b^{3}\right)+\frac{m}{2}\left(y^{2}-b^{2}\right) \\
=\int_{0}^{b} f(r) r^{2} d r-\int_{0}^{y(t)} u(r, t) r^{2} d r .
\end{aligned}
$$

Now we will use this formula in order to prove the following lemma containing the case of $b_{1}=b_{2}$ excluded in Lemma 3 .

Lemma $3^{\prime}$. If $b_{1} \leq b_{2}$ and $f_{1} \leq f_{2}$, then

$$
y_{1}(t) \leq y_{2}(t) \quad(t \geq 0)
$$

Proof. In view of Lemma 3 we have only to prove it for the case of $b_{1}=b_{2}$. Then we take an arbitrary positive constant $\delta$, and consider three pairs of data $\left(b_{2}, f_{1}\right),\left(b_{2}, f_{2}\right)$ and $\left(b_{2}+\delta, f_{2}\right)$ (where $f_{2}$ is taken to be zero for $\left.b_{2}<r \leq b_{2}+\delta\right)$ and the corresponding solutions $\left(y_{1}, u_{1}\right)$, $\left(y_{2}, u_{2}\right)$ and $\left(y_{2}^{\delta}, u_{2}^{\delta}\right)$. We have from Lemma 3

$$
y_{1}<y_{2}^{o}, \quad y_{2}<y_{2}^{o} \quad(t \geq 0) .
$$

By applying the fundamental formula (2.2) to the two solutions $\left(y_{2}^{\delta}, u_{2}^{\delta}\right)$ and $\left(y_{2}, u_{2}\right)$ respectively, we get

$$
\frac{1}{3}\left(\frac{1}{\alpha}-\frac{m}{b_{2}+\delta}\right)\left(y_{2}^{\delta}\right)^{3}+\frac{m}{2}\left(y_{2}^{\delta}\right)^{2}
$$




$$
\begin{aligned}
= & \frac{1}{3}\left(\frac{1}{\alpha}-\frac{m}{b_{2}+\delta}\right)\left(b_{2}+\delta\right)^{3}+\frac{m}{2}\left(b_{2}+\delta\right)^{2} \\
& +\int_{0}^{\left.b_{2}\right\urcorner \delta} f_{2}(r) r^{2} d r-\int_{0}^{y_{2}^{\delta}(t)} u_{2}^{\delta}(r, t) r^{2} d r
\end{aligned}
$$

and

$$
\begin{aligned}
\frac{1}{3}\left(\frac{1}{\alpha}-\frac{m}{b_{2}}\right) y_{2}{ }^{3}+\frac{m}{2} y_{2}{ }^{2} \\
=\frac{1}{3}\left(\frac{1}{\alpha}-\frac{m}{b_{2}}\right) b_{2}{ }^{3}+\frac{m}{2} b_{2}{ }^{2} \\
\quad+\int_{0}^{b_{2}} f_{2}(r) r^{2} d r-\int_{0}^{y_{2}(t)} u_{2}(r, t) r^{2} d r .
\end{aligned}
$$

By subtracting the latter equation from the former we have

(2. 4) $\frac{1}{3}\left(\frac{1}{\alpha}-\frac{m}{b_{2}}\right)\left[\left(y_{2}^{o}\right)^{3}-y_{2}{ }^{3}\right]+\frac{m}{2}\left[\left(y_{2}^{o}\right)^{2}-y_{2}{ }^{2}\right]$

$$
\begin{aligned}
= & \frac{1}{3}\left(\frac{m}{b_{2}+\delta}-\frac{m}{b_{2}}\right)\left(y_{2}^{\delta}\right)^{3}+\frac{1}{3}\left(\frac{1}{\alpha}-\frac{m}{b_{2}}\right)\left[\left(b_{2}+0\right)^{3}-b_{2}{ }^{3}\right] \\
& +\frac{1}{3}\left(\frac{m}{b_{2}}-\frac{m}{b_{2}+\delta}\right)\left(b_{2}+\delta\right)^{3}+\frac{m}{2}\left[\left(b_{2}+\delta\right)^{2}-b_{2}{ }^{2}\right] \\
& -\int_{0}^{y_{2}(t)}\left[u_{2}^{o}(r, t)-u_{2}(r, t)\right] r^{2} d r-\int_{y_{2}(t)}^{y_{2}{ }^{o}(t)} u_{2}^{o}(r, t) r^{2} d r .
\end{aligned}
$$

It is known from the maximum principle that

$$
u_{2}^{o}(r, t) \geq 0 \quad\left(0 \leq r \leq y_{2}(t), t \geq 0\right)
$$

and

$$
u_{2}^{\delta}(r, t)-u_{2}(r, t) \geq 0 \quad\left(0 \leq r \leq y_{2}(t), t \geq 0\right) .
$$

By applying these inequalities in (2.4) we get

(2.5) $\frac{1}{3}\left(\frac{1}{\alpha}-\frac{m}{b_{2}}\right)\left(y_{2}^{o}\right)^{3}+\frac{m}{2}\left(y_{2}^{\delta}\right)^{2}$

$$
\begin{aligned}
\leq & \frac{1}{3}\left(\frac{1}{\alpha}-\frac{m}{b_{2}}\right) y_{2}{ }^{3}+\frac{m}{2} y_{2}{ }^{2}-\frac{m \delta}{3 b_{2}\left(b_{2}+\delta\right)}\left(y_{2}{ }^{o}\right)^{3} \\
& +\frac{1}{3}\left(\frac{1}{\alpha}-\frac{m}{b_{2}}\right)\left[\left(b_{2}+\delta\right)^{3}-b_{2}{ }^{3}\right]-\frac{m \delta}{3 b_{2}}\left(b_{2}+\delta\right)^{2} \\
& +\frac{m}{2}\left[\left(b_{2}+\delta\right)^{2}-b_{2}{ }^{2}\right] .
\end{aligned}
$$


Here we note that the function

$$
F(y)=\frac{1}{3}\left(\frac{1}{\alpha}-\frac{m}{b}\right) y^{3}+\frac{m}{2} y^{2}
$$

is monotone increasing for all $y>0$ if $b>\alpha^{\prime} m$, and also for $0<y<\frac{m b}{\alpha m-b}$ if $b<\alpha m$, and that by the continuity and the monotonicity (Lemma 2) of $y_{2}(t)$ and $y_{1}^{\prime}(t)$

$$
b_{2}+o \leq y_{2}{ }^{\circ}(t) \quad\left(\leq \frac{m b_{2}}{m-b_{2}}, \quad \text { if } \quad b_{2}<\alpha m\right)
$$

and

$$
b_{1}=b_{2} \leq y_{1}(t) \quad\left(\leq \frac{m b_{2}}{m-b_{2}}, \quad \text { if } \quad b_{2}<\alpha m\right)
$$

hold for sufficiently small $t$ and $\delta$. Hence, from (2.3), (2.5) we get

$$
\frac{1}{3}\left(\frac{1}{\alpha}-\frac{m}{b_{2}}\right) y_{1}^{3}+\frac{m 2}{2} y_{1}^{2} \leq \frac{1}{3}\left(\frac{1}{\alpha}-\frac{m}{b_{2}}\right) y_{2}^{3}+\frac{m}{2} y_{2}^{2}+O(0)
$$

for sufficiently small $t$ and $\delta$. The last term $O(\delta)$ tends to zero as $\delta \rightarrow 0$ since $y_{2}^{o}(t)$ is uniformly bounded for small $t$ and $\delta$. Since $\delta$ is arbitrary, it follows from the last inequality that

$$
\frac{1}{3}\left(\frac{1}{\alpha}-\frac{m}{b_{2}}\right) y_{1}^{3}+\frac{m}{2} y_{1}{ }^{2} \leq \frac{1}{3}\left(\frac{1}{\alpha}-\frac{m}{b_{2}}\right) y_{2}{ }^{3}+\frac{m}{2} y_{2}{ }^{2}
$$

for sufficiently small $l$. Again by the above note we get

$$
y_{1}(t) \leq y_{2}(t) \text { for sufficiently small } t \text {. }
$$

If $f_{1} \equiv f_{2}$ and $b_{1}=b_{2}$, we have also $y_{1}(t) \geq y_{2}(t)$, and hence $y_{1}(t)$ $=y_{2}(t)$ for sufficiently small $t$ because both $y_{1}(t)$ and $y_{2}(t)$ are bounded for all $t>0$ that is to be shown in Lemma 4 . Continuing the same reasoning for each small time interval successively, we get $y_{1}(t)=y_{2}^{\prime}(t)$ for all $t>0$.

Next we suppose that $f_{1} \not \equiv f_{2}, f_{1} \leq f_{2}$ and $b_{1}=b_{2}$. If the equality $y_{1}(t)$ $=y_{2}(t)$ holds for an interval $0 \leq t \leq t_{0}$, the strong maximum principle yields

$$
u_{1}(r, l)-u_{2}(r, l)<0 \text { for } \quad 0 \leq r \leq l_{1}(l), 0<l \leq l_{0} .
$$

Hence, from the condition that $u_{1}\left(\prime_{1}^{\prime}(t), \iota\right)-u_{2}\left(y_{1}(t), t\right)=0$ we get by 
Friedman's lemma

$$
\frac{\partial u_{1}}{\partial r}\left(y_{1}(t), t\right)-\frac{\partial u_{2}}{\partial r}\left(y_{1}(t), t\right)>0 \text { i.e. } \dot{y}_{1}(t)<\dot{y}_{2}(t) \text { for } 0<t \leq t_{0} .
$$

But this is a contradiction to our assumption. Therefore we get $y_{1}(t)<$ $y_{2}(t)$ from (2.6), and also $u_{1}(r, t)<u_{2}(r, t)$ for some sufficiently small interval $0<t \leq t_{0}$ and $0 \leq r<y_{1}(t)$. By using Lemma 3 (its modified one for the latter time $t \geq t_{0}$ ) we have again

$$
y_{1}(t)<y_{2}(t) \text { for all } t>0 \text {. }
$$

Thus we have proved Lemma $3^{\prime}$.

Lemma 4. Suppose that there exists a solution $(y, u)$ of the problem (P) Sor all $t>0$. Then $y(t)$ is bounded and

$$
\lim _{t \rightarrow \infty} y(t)=y(\infty)
$$

exists, where $y(\infty)$ is determined from the equation (2.7) below.

Proof. Let us consider the solution $w(r, t)$ of the problem

$$
\begin{aligned}
& \frac{\partial w}{\partial t}-\Delta w=0 \quad(r>0, t>0), \\
& w(r, 0)= \begin{cases}f(r)-\left(\frac{m}{b}-\frac{m}{r}\right) & (0<r<b), \\
0 & (r>b) .\end{cases}
\end{aligned}
$$

It is clear that $u(r, t)-\left(\frac{m}{b}-\frac{m}{r}\right)(\geq 0)$ is bounded from above by $w(r, t)$. However we see that for sufficiently large $t$

$$
\begin{aligned}
r^{2} w(r, t) & =r \int_{0}^{b} g(r, t ; \xi, 0)\left\{f(\xi)-\left(\frac{m}{b}-\frac{m}{\xi}\right)\right\} \xi d \xi \\
& <\frac{1}{2 \sqrt{\pi} t^{3 / 2}} r^{2} \exp \left(-\frac{(r-b)^{2}}{4 t}\right) \int_{0}^{b}\left\{f(\xi)-\left(\frac{m}{b}-\frac{m}{\xi}\right)\right\} \xi^{2} d \xi \\
& <\frac{b}{2 \sqrt{\pi} t^{3 / 2}}\left(\frac{b+\sqrt{b^{2}+12 t}}{2}\right)^{2} \max _{0 \leq r \leq b} r^{2}\left\{f(r)-\left(\frac{m}{b}-\frac{m}{r}\right)\right\}
\end{aligned}
$$

where $g$ is the Green's function defined in $\S 1$. Hence we see that $\lim _{t \rightarrow \infty} r^{2} w(r, t)=0$ uniformly and further 


$$
\lim _{t \rightarrow \infty} r^{2}\left\{u(r, t)-\left(\frac{m}{b}-\frac{m}{r}\right)\right\}=0 \quad \text { uniformly. }
$$

We take a positive constant $\varepsilon$ less than $b^{2} / 3 \alpha$. Then there is a $T_{0}$ such that

$$
\left|r^{2} u(r, t)-r^{2}\left(\frac{m}{b}-\frac{m}{r}\right)\right|<\varepsilon \quad \text { for } t>T_{0}
$$

Hence

$$
-\int_{0}^{y(t)} r^{2} u(r, t) d r<\varepsilon y(t)-\frac{m y^{3}}{3 b}+\frac{m y^{2}}{2} \text { for } t>T_{0}
$$

By applying the last inequality in (2.2) we get

$$
\frac{y(t)^{3}}{3 \alpha}-\varepsilon y(t)<\frac{b^{3}}{3}\left(\frac{1}{\alpha}-\frac{m}{b}\right)+\frac{m b^{2}}{2}+\int_{0}^{b} f(r) r^{2} d r \quad \text { for } t>T_{0} .
$$

Since $\frac{y^{3}}{3 \alpha}-\varepsilon y>\frac{b^{3}}{3 \alpha}-\varepsilon b>0$ (from Lemma 2 and our assumption of $\varepsilon$ ) it follows that $y(t)$ is bounded for all $t>T_{0}$, and hence for all $t>0$ because $y(t)$ is continuous for $t \geq 0$. In addition $y(t)$ is monotone increasing from Lemma 2. Thus there exists a limit

$$
\lim _{t \rightarrow \infty} y(t)=y(\infty)
$$

By taking $t \rightarrow \infty$ in (2.2) we get

$$
\frac{1}{3 \alpha} y(\infty)^{3}=\frac{1}{3 \alpha} b^{3}+\frac{m}{6} b^{2}+\int_{0}^{b} f(r) r^{2} d r .
$$

Thus we have proved Lemma 4.

Next we assume, in addition to (2.1), that

$$
f(b)=0 \text {. }
$$

Then there is a positive constant $N$ such that

$$
0 \leq f(r) \leq N\left(\frac{1}{r}-\frac{1}{b}\right) \quad(0<r \leq b) .
$$

Lemma 5. Under the assumptions (2.1), (2.8) (and also (2.9)),

$$
0 \leq \frac{\dot{y}(\iota)}{\alpha}=-u_{r}(y(t), \iota) \leq \frac{N}{y(t)^{2}} \quad(t>0) .
$$


Proof. Introduce a 'barrier' function

$$
\omega_{t_{0}}(r, t)=N\left[\frac{1}{r}-\frac{1}{y\left(t_{0}\right)}\right]+\frac{m}{b}-\frac{m}{y\left(t_{0}\right)}
$$

depending on a parameter $t_{0}$. It satisfies the basic equation $(\mathrm{P}-1)$ and the following inequalities:

$$
\begin{gathered}
\omega_{t_{0}}(y(t), t)=N\left[\frac{1}{y(t)}-\frac{1}{y\left(t_{0}\right)}\right]+\frac{m}{b}-\frac{m}{y\left(t_{0}\right)}>\frac{m}{b}-\frac{m}{y(t)} \quad\left(0 \leq t \leq t_{0}\right), \\
\omega_{t_{0}}(r, 0)=N\left[\frac{1}{r}-\frac{1}{y\left(t_{0}\right)}\right]+\frac{m}{b}-\frac{m}{y\left(t_{0}\right)}>N\left[\frac{1}{r}-\frac{1}{y\left(t_{0}\right)}\right] \\
>N\left[\frac{1}{r}-\frac{1}{b}\right] \geq f(r) \quad(0<r \leq b) .
\end{gathered}
$$

Then it follows from the maximum principle that

$$
\omega_{\iota_{0}}(r, t)-u(r, t) \geq 0 \quad\left(0 \leq r \leq y(t), 0 \leq t \leq t_{0}\right),
$$

and hence that

$$
\frac{u\left(y\left(t_{0}\right)-\rho, t\right)-\left(\frac{m}{b}-\frac{m}{y\left(t_{0}\right)}\right)}{\rho} \leq \frac{N}{\left(y\left(t_{0}\right)-\rho\right) y\left(t_{0}\right)}
$$

for any sufficiently small $\rho$. This yields

$$
-\frac{\partial u}{\partial r}\left(y\left(t_{0}\right), t_{0}\right) \leq \frac{N}{y\left(t_{0}\right)^{2}}
$$

Since $t_{0}$ is arbitrary, we find also that the right inequality of (2.10) is true. The left one is so by Lemma 2. Thus the proof of Lemma 5 is completed.

\section{$\S 3$. Uniqueness and Existence of the Solution}

The uniqueness theorem follows directly from Lemma $3^{\prime}$. Indeed we suppose that there are two solutions $\left(y_{i}, u_{i}\right)(i=1,2)$. Then $y_{1} \equiv y_{2}$ follows from Lemma $3^{\prime}$ and hence $u_{1} \equiv u_{2}$ follows from the well known theory of the problem with the prescribed boundary.

Theorem 1. The sy'stem (P) has not more than one solution under the condition (2.1). 
Next we will show existence of the solution by constructing it. For that we consider an auxiliary problem, which we call (AP), with the prescribed boundary $r=y_{0}(t)$ :

$$
(\mathrm{AP}) \begin{cases}\frac{\partial u}{\partial t}=\Delta u & \left(0 \leq r \leq y_{0}(t), t>t_{0}\right) \\ u\left(y_{0}(t), t\right)=\frac{m}{b}-\frac{m}{y_{0}(t)} & \left(t>t_{0}\right) \\ u\left(r, t_{0}\right)=f_{0}(r) & \left(0 \leq r \leq y_{0}\left(t_{0}\right)\right)\end{cases}
$$

For each $\theta, 0<\theta<b$, we construct an approximate solution $\left(y^{\theta}, u^{\theta}\right)$ of the problem $(\mathrm{P})$ by retarding the argument

$$
\dot{y}(t)=-\alpha \frac{\partial u}{\partial r}(y(t-\theta), t-\theta)
$$

in the Stefan's condition $(\mathrm{P}-3)$. In the first interval $0 \leq t \leq \theta$ we set $v^{\theta}(t) \equiv b$ and define $\imath^{\theta}(r, t)$ to be the unique solution of (AP) with $t_{0}=0$, in which $y_{0}^{\prime}(t)$ and $f_{0}(r)$ have been replaced by $y^{\theta}(t)$ and $f(r)$, respectively. It is easy to verify that, due to our choice of $y^{\theta}(t)$ and $f(r)$ (satisfying (2.1), (2.8) $((2.9))), \frac{\partial u^{\theta}}{\partial r}(b, t)$ exists and is continuous for $0 \leq t \leq \theta$. Moreover, by the similar way to the proof of Lemma 5 , we have

$$
0 \leq-\frac{\partial u^{\theta}}{\partial r}(b, t) \leq \frac{N}{b^{2}}
$$

Now we proceed by induction. Assume that $\left(y^{0}, u^{0}\right)$ has been constructed for $0 \leq t \leq n \theta$, that $y^{\theta}(t)$ is continuously differentiable for $\theta \leq t \leq n \theta$, that $\partial u^{\theta} /(\partial r)\left(y^{\theta}(t), t\right)$ exists and is continuous, that

$$
0 \leq-\frac{\partial u^{\theta}}{\partial r}\left(y^{\theta}(t), t\right) \leq \frac{N}{v^{\theta}(t-\theta)^{2}},
$$

and that

$$
y^{\theta}(t)=b-\alpha \int_{\theta}^{t} \frac{\partial u^{\theta}}{\partial r}\left(\nu^{\theta}(\tau-\theta), \tau-\theta\right) d \tau \text { for } t \geq \theta
$$

In the next step $n \theta \leq t \leq(n+1) \theta$ we define $y^{0}(t)$ by (3.2) and solve (AP) with $t_{0}=n \theta, y_{0}(t)=y^{\theta}(t), f_{0}(r)=u^{0}(r, n \theta)$ for $u^{\theta}(r, t)$ up one more step into the region $0 \leq r \leq y(t), n \theta \leq t \leq(n+1) \theta$. By the inductive 
hypothesis on $\frac{\partial u^{\theta}}{\partial r}, y^{0}(t)$ is a continuously differentiable function satisfying

$$
0 \leq \dot{y}^{\theta}(t) \leq \frac{N \alpha}{y^{\theta}(t-\theta)^{2}}
$$

Hence $\frac{\partial u^{\theta}}{\partial r}\left(y^{\theta}(t), t\right)$ exists and is continuous, and from the same argument as that in the proof of Lemma 5 , we have

$$
0 \leq-\frac{\partial u}{\partial r}\left(y^{\theta}(t), t\right) \leq \frac{N}{y^{\theta}(t-\theta)^{2}} .
$$

Thus the approximate solution $\left(y^{0}, u^{\theta}\right)$ can be constructed throughout the interval $0 \leq t \leq T$, and $y^{\theta}(t)$ satisfies (3.3) for the same interval (in which $y^{\theta}(t-\theta)$ is taken to be equal to $b$ for $0 \leq t \leq \theta$ ).

Hence the functions $\left\{y^{\theta}(t)\right\}$ form an equicontinuous uniformly bounded family in $0 \leq t \leq T$. We choose a sequence of $\theta$ 's tending to zero. By Ascoli-Arzela's theorem there is a subsequence, denoted by $v^{\theta}(t)$, that converges uniformly to a monotone Lipschitz continuous function $y(t)$. Let $u(r, t)$ be the unique solution of (AP) for the choice of $t_{0}=0$, $f_{0}(r)=f(r)$ and $y_{0}(t)=y(t)$. Given any $\varepsilon>0$ we have, for $0 \leq t \leq T$ and all $\theta$ 's of the subsequence that are sufficiently small,

$$
\left|y^{\theta}(t)-y(t)\right| \leq \frac{b^{2} \varepsilon}{N}=\rho
$$

For convenience we extend the functions $u^{0}$ and $u$ by setting them equal to $\frac{m}{b}-\frac{m}{y(t)}$ for each $t$ outside their natural domains of definition. Then in any region $0 \leq r \leq \max \left(y^{\theta}(t), y(t)\right), 0 \leq t \leq T$, the difference $u^{\theta}-u$ is bounded, using the maximum principle and Lemma 5 , by

$$
\left|u^{\theta}(r, t)-u(r, t)\right| \leq \frac{N \rho}{b^{2}}=\varepsilon
$$

Thus the corresponding subsequence $u^{\theta}$ converges uniformly to $u$.

The pair of functions $(y, u)$ defined as above, of course, satisfies the conditions $(\mathrm{P}-1),(\mathrm{P}-2),(\mathrm{P}-4)$ and $(\mathrm{P}-5)$. It remains to assure $(\mathrm{P}-3)$. We note that $(\mathrm{P}-3)$ is equivalent to the fundamental formula (2.2) under the condition of Lipshitz continuity of $y(t)$. For $\left(y^{\theta}, u^{\theta}\right)$ we have by the same way as in $\S 2$ 


$$
\begin{aligned}
& \int_{\theta}^{t} y^{\theta}(\tau)^{2}\left(-\frac{1}{\alpha} \dot{y}^{\theta}(\tau+\theta)\right) d \tau-\int_{0}^{y^{\theta}(t)} u^{\theta}(r, t) r^{2} d r \\
& \quad+\int_{0}^{b} u^{\theta}(r, \theta) r^{2} d r+\int_{\theta}^{t}\left(\frac{m}{b}-\frac{m}{y^{\theta}(\tau)}\right) y^{\theta}(\tau)^{2} \dot{y}^{\theta}(\tau) d \tau=0 .
\end{aligned}
$$

The first and the last integrals of the left-hand side may be written as

$$
\begin{gathered}
-\frac{1}{\alpha} \int_{\theta}^{t} y^{\theta}(\tau+\theta)^{2} \dot{y}^{\theta}(\tau+\theta) d \tau+\frac{1}{\alpha} \int_{\theta}^{t}\left(y^{\theta}(\tau+\theta)^{2}-y^{\theta}(\tau)^{2}\right) \dot{y}^{\theta}(\tau+\theta) d \tau \\
=-\frac{1}{3 \alpha}\left(y^{\theta}(t+\theta)^{3}-y^{\theta}(2 \theta)^{3}\right)+O(\theta)
\end{gathered}
$$

and

$$
\begin{aligned}
\int_{\theta}^{t}\left(\frac{m}{b}\right. & \left.-\frac{m}{y^{\theta}(\tau)}\right) y^{\theta}(\tau)^{2} \dot{y}^{\theta}(\tau) d \tau \\
& =\frac{m}{3 b}\left(y^{\theta}(t)^{3}-y^{\theta}(\theta)^{s}\right)-\frac{m}{2}\left(y^{\theta}(t)^{2}-y^{\theta}(\theta)^{2}\right) .
\end{aligned}
$$

Hence, by taking the limit in (3.4) as the subsequence $\theta$ tends to zero, it follows from the uniform convergence of $y^{\theta}$ to $y$ and $u^{\theta}$ to $u$ that $(y, u)$ satisfies $(2.2)$. Therefore $(y, u)$ is actually a solution to the problem $(\mathrm{P})$ and $y(t)$ is continuously differentiable for $0<t \leq T$. Thus we have proved the existence of the solution under the assumptions (2.1) and (2.8).

We can, furthermore, show that only the assumption (2.1) is sufficient for the existence. Indeed we have

Theorem 2. Suppose that $f(r)$ is smooth except for finitely many points of jump discontinuity where both $f^{\prime}(r-0)$ and $f^{\prime}(r+0)$ exist, and satisfies (2.1). Then there exists a solution $(y, u)$ of the problem $(\mathrm{P})$.

Proof. Define

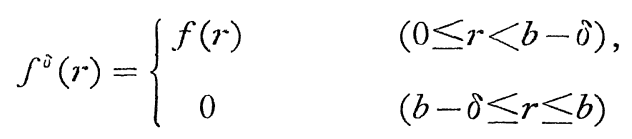

for each $\delta(0<\delta<b)$ Since $f$ is bounded in $0 \leq r \leq b$, there exists an 
$N(\delta)$ for each $\delta$ such that

$$
0 \leq f^{\delta}(r) \leq N(\delta)\left(\frac{1}{r}-\frac{1}{b}\right)
$$

It follows from the above discussion that there exists a unique solution $\left(y^{o}, u \iota^{o}\right)$ of the problem in which $f$ is replaced by $f^{o}$ for each $\delta$. We see, from Lemma $3^{\prime}$, that $y^{\delta_{1}} \leq y^{\delta_{2}}$ holds for $\delta_{2}<\delta_{1}$. Choose a sequence of $\delta_{n}$ $(n=1,2,3, \cdots)$ tending to zero. Then the corresponding $y^{\hat{o}_{n}} \quad(n=1,2,3$, $\cdots)$ is a monotone increasing sequence bounded from above by, for example, the free boundary $y$ of the solution of $(\mathrm{P})$ in which $b$ is replaced by $b+1$ and $f$ is extended as zero for $b<r \leq b+1$. Hence $y^{\hat{o}_{n}}(t)$ converges to a limit $y(t)$ at each time $t$ as $n \rightarrow \infty$. We shall show that $y(t)$ is a continuous function in $0 \leq t<\infty$. Since $y^{\delta_{1}}(t)$ is strictly monotone increasing in $t$ (via Lemma 2), $y^{\hat{o}_{1}}(\sigma)-b$ is strictly positive for any $\sigma>0$, and hence $y^{\delta_{n}}(\sigma)-b>y^{1}(\sigma)-b>0$. By applying Lemma 5 to the solution $\left(y^{\delta_{n}}, u u^{\delta_{n}}\right)$ in the restricted region $\left\{b<r<y^{\delta_{n}}(t), t>\sigma\right\}$, we find that there is a positive constant $\kappa_{\sigma}$ (playing the role of $N$ in Lemma 5) depending on any fixed bounds of $f$ and $y^{1}(\sigma)-b$ such that

$$
0 \leq \frac{\dot{y}^{n}(t)}{\alpha}=-\frac{\partial u^{\delta_{n}}}{\partial r}\left(y^{\partial_{n}}(t), t\right) \leq \frac{\kappa_{\sigma}}{y^{n}(t)^{2}} \leq \frac{\kappa_{\sigma}}{b^{2}} \quad(t \geq \sigma)
$$

for all $n$. Hence all of $y^{\hat{o}_{n}}(t)$ are Lipshitz continuous with the uniform Lipshitz constant $\frac{\kappa_{\sigma}}{b^{2}}$ in $t \geq \sigma$, and therefore the limit function $y(t)$ also is Lipshitz continuous with the same constant for any $t \geq \sigma$. Next, in order to show the continuity of $y(t)$ at $t=0$, we consider the following auxiliary problem for finding $(\rho, v)$ :

$$
\begin{cases}\frac{\partial v}{\partial t}-\Delta u=0 & (b<r<\rho(t), t>0) \\ v(b, t)=M & (t>0) \\ v(\rho(t), t)=\frac{m}{b}-\frac{m}{\rho(t)} & (t>0) \\ \dot{\rho}(t)=-\alpha \frac{\partial v}{\partial r}(\rho(t), t) & (t>0) \\ \rho(0)=b & \end{cases}
$$

where $M=\max \left(\max _{0 \leq r \leq b} f(r), \frac{m}{b}\right)$. It shall be shown in $\S 4$ that there is 
a unique solution of (3.5) and $\rho(t)$ is a monotone increasing function for all $t \geq 0$. And we find, by the same argument as in the proofs of Lemma 3 and $3^{\prime}$, that $.^{y^{\delta_{n}}}(t) \leq \rho(t)$ holds for all $t>0$. Hence we get $y^{\prime}(t) \leq p(t)$ for all $t \geq 0$. Because both $y^{\delta_{1}}(t)$ and $\rho(t)$ are continuous for $t \geq 0, y^{\hat{o}_{1}}(0)=\rho(0)=b$ and $y^{\hat{o}_{1}}(t) \leq y(t) \leq \rho(t), v(t)$ also is continuous at $t=0$. Denote by $u$ the unique solution of the ordinary problem $(\mathrm{P}-1)$, $(\mathrm{P}-2),(\mathrm{P}-5)$ with the prescribed boundary $y(t)$. From the maximum principle we find also that $u^{\iota_{n}}(r, t)$ converges uniformly to $u(r, t)$ as $n \rightarrow \infty$. Hence it is easily seen that $(y, u)$ also satisfies the fundamental formula (2.2), therefore $y(t)$ is continuously differentiable for $t>0$, and that $(y, u)$ is nothing but a solution of $(\mathrm{P})$.

\section{$\S$ H. Comment on the Unique Solvability of the Problem (3.5)}

We consider the following problem

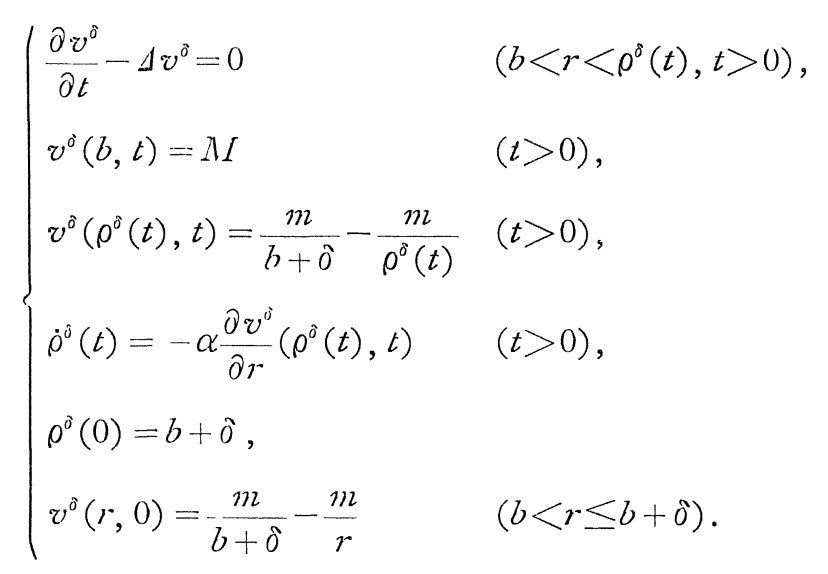

It has a unique solution $\left(\rho^{\hat{o}}, v^{\delta}\right)$. First we will show it. By repeating almost the same argument as that in the proof of Lemma 1, 2, we get

$$
\dot{\rho}^{\grave{o}}(t) \geq 0 \quad(t>0)
$$

and, by using the function

$$
\omega_{t_{0}}(r, t)=\frac{M}{\frac{1}{b}-\frac{1}{\rho^{\hat{o}}\left(t_{0}\right)}}\left\{\frac{1}{r}-\frac{1}{\rho^{\hat{o}}\left(t_{0}\right)}\right\}+\frac{m}{b+\delta}-\frac{m}{\rho^{\hat{\delta}}\left(t_{0}\right)}
$$

as a 'barrier' to $v^{\circ}$, we get 
(4. 3) $0 \leq \frac{\dot{\rho}^{\delta}(t)}{\alpha}=-\frac{\partial v^{\delta}}{\partial r}\left(\rho^{\hat{\delta}}(t), t\right) \leq \frac{M b}{\rho^{\delta}(t)\left(\rho^{\delta}(t)-b\right)} \leq \frac{M}{\rho^{\delta}(t)-b}$,

through the analogous argument to that for the proof of Lemma 5. Since $\frac{\partial v^{\delta}}{\partial r}(b, t) \leq 0$ (by the maximum principle) we have

$$
\frac{\partial v^{\delta}}{\partial r}(r, t) \leq 0 \quad\left(b \leq r \leq \rho^{\delta}(t), t>0\right)
$$

as in the proof of Lemma 2. Hence we get an inequality corresponding to that in Lemma 3

$$
\rho^{\delta_{1}}(t)<\rho^{\delta_{2}}(t) \quad\left(\delta_{1}<\delta_{2}, t \geq 0\right) .
$$

It also will be found that

$$
\rho^{\delta_{1}}(t)=\rho^{\delta_{2}}(t) \quad\left(\delta_{1}=\delta_{2}, t \geq 0\right) .
$$

In fact we consider Green's formula

$$
\oint r^{2} u v^{\delta} d r+\left(r^{2} u \frac{\partial v^{\delta}}{\partial r}-r^{2} v^{\delta} \frac{\partial u}{\partial r}\right) d \tau=0
$$

where $u \equiv \frac{1}{b}-\frac{1}{r}$, and the integration is done along the closed boundary of the region $\left\{b<r<\rho^{\delta}(t), 0<\tau<t\right\}$. It is described explicitly as

$$
\begin{aligned}
\frac{1}{3 b}\left(\frac{1}{\alpha}-\frac{m}{b+\delta}\right)\left\{\rho^{\delta}(t)^{3}-(b+\delta)^{3}\right\} \\
\quad-\frac{1}{2}\left\{\frac{1}{\alpha}-\left(\frac{m}{b}+\frac{m}{b+\delta}\right)\right\}\left\{\rho^{\delta}(t)^{2}-(b+\delta)^{2}\right\}-m\left\{\rho^{\delta}(t)-(b+\delta)\right\} \\
=m \int_{0}^{t} \frac{d \tau}{\rho^{\delta}(\tau)}-\int_{b}^{\rho(t)} r^{2}\left(\frac{1}{b}-\frac{1}{r}\right) v^{\delta}(r, t) d r+\left(M-\frac{m}{b+\delta}\right) t \\
\quad+\int_{b}^{b+\delta} r^{2}\left(\frac{1}{b}-\frac{1}{r}\right)\left(\frac{m}{b+\delta}-\frac{m}{r}\right) d r
\end{aligned}
$$

By subtracting the last formula with $\delta=\delta_{1}$ from that with $\delta=\delta_{2} \quad\left(\delta_{1}<\delta_{2}\right)$ we have

$$
\begin{aligned}
& \frac{1}{3 b}\left(\frac{1}{\alpha}-\frac{m}{b+\delta_{1}}\right) \rho^{\delta_{2}}(t)^{3}-\frac{1}{2}\left\{\frac{1}{\alpha}-\left(\frac{m}{b}+\frac{m}{b+\delta_{1}}\right)\right\} \rho^{\delta_{2}}(t)^{2}-m \rho^{\delta_{2}}(t) \\
& =\frac{1}{3 b}\left(\frac{1}{\alpha}-\frac{m}{b+\delta_{1}}\right) \rho^{\delta_{1}}(t)^{3}-\frac{1}{2}\left\{\frac{1}{\alpha}-\left(\frac{m}{b}+\frac{m}{b+\delta_{1}}\right)\right\} \rho^{\delta_{1}}(t)^{2}-m \rho^{\delta_{1}}(t)
\end{aligned}
$$




$$
\begin{aligned}
& -\int_{b}^{\rho^{\delta_{1}}(t)}\left(\frac{1}{b}-\frac{1}{r}\right)\left(v^{\hat{\delta}_{2}}(r, t)-v^{\delta_{1}}(r, t)\right) r^{2} d r \\
& -\int_{\rho^{\delta_{1}}(t)}^{\rho_{\delta_{2}}(t)}\left(\frac{1}{b}-\frac{1}{r}\right) v^{\hat{\delta}_{2}}(r, t) r^{2} d r+m \int_{0}^{t}\left(\frac{1}{\rho^{\delta_{2}}(\tau)}-\frac{1}{\rho^{\delta_{1}}(\tau)}\right) d \tau \\
& +O\left(\delta_{2}-\delta_{1}\right) .
\end{aligned}
$$

Since $v^{\hat{\delta}_{2}}(r, t)-v^{\hat{o}_{1}}(r, t)>0$ for $0<r<\rho^{\delta_{1}}(t), \quad v^{\hat{o}_{2}}(r, t)>0$ and $\rho^{\hat{o}_{2}}(t)>$ $\rho^{\delta_{1}}(t)$, we get

$$
\begin{aligned}
\frac{1}{3 b}\left(\frac{1}{\alpha}-\right. & \left.\frac{m}{b+\delta_{1}}\right) \rho^{\delta_{2}}(t)^{3}-\frac{1}{2}\left\{\frac{1}{\alpha}-\left(\frac{m}{b}+\frac{m}{b+\bar{\delta}_{1}}\right)\right\} \rho^{\delta_{2}}(t)^{2}-m \rho^{\delta_{2}}(t) \\
\leq & \frac{1}{3 b}\left(\frac{1}{\alpha}-\frac{m}{b+\delta_{1}}\right) \rho^{\delta_{1}}(t)^{3}-\frac{1}{2}\left\{\frac{1}{\alpha}-\left(\frac{m}{b}+\frac{m}{b+\delta_{1}}\right)\right\} \rho^{\delta_{1}}(t)^{2}-m \rho^{\delta_{1}}(t) \\
& +O\left(\delta_{2}-\delta_{1}\right) .
\end{aligned}
$$

Because the function

$$
F(\rho) \equiv \frac{1}{3 b}\left(\frac{1}{\alpha}-\frac{m}{b+\delta_{1}}\right) \rho^{3}-\frac{1}{2}\left\{\frac{1}{\alpha}-\left(\frac{m}{b}+\frac{m}{b+\delta_{1}}\right)\right\} \rho^{2}-m \rho
$$

is monotone increasing for $\rho>b$ if $\rho-b$ is small, we find that

$$
\rho^{\delta_{2}}(t) \leq \rho^{\delta_{1}}(t)+O\left(\delta_{2}-\delta_{1}\right) \text { for small } t
$$

Hence and from (4.5), by tending $\delta_{2}$ to $\hat{\delta}_{1}$ infinitely, we obtain (4.6) for small $t$, and hence also for all $t$.

At this stage, by using the a priori estimates (4.2) (4.5), we can construct a solution $\left(\rho^{\delta}, v^{\delta}\right)$ in the same way as in $\S 3$. And this solution, by (4.6), turns out to be unique.

Next we will show that there are two positive constants $\delta_{1}$ and $\delta_{2}$ such that

$$
b+\delta_{1} \sqrt{t}<\rho^{\delta}(t)<b+\delta+\delta_{2} \sqrt{t} \quad(0 \leq t \leq \varepsilon) .
$$

Put

$$
r\left[v^{\delta}-\left(\frac{m}{b+\delta}-\frac{m}{r}\right)\right]=r v^{\delta}
$$

Then $w^{\delta}$ satisfies the following equations: 


$$
\left\{\begin{array}{l}
\frac{\partial w^{\delta}}{\partial t}-\frac{\partial^{2} w^{\delta}}{\partial r^{2}}=0 \quad\left(b<r<\rho^{\delta}(t), t>0\right), \\
w^{\delta}(b, t)=b\left[M+\frac{\delta}{b(b+\delta)}\right] \\
w^{\delta}\left(\rho^{\delta}(t), t\right)=0, \\
\rho^{\delta}(t) \dot{\rho}^{\delta}(t)+\frac{\alpha m}{\rho^{\delta}(t)}=-\alpha \frac{\partial w^{\delta}}{\partial r}\left(\rho^{\delta}(t), t\right) \quad(t>0), \\
\rho^{\delta}(0)=b+\delta \\
w^{\delta}(r, 0)=0 .
\end{array}\right.
$$

We consider, in addition to $w^{\delta}$, the function

$$
w(r, t)=\frac{2 b}{\alpha} \sum_{n=1}^{\infty} \frac{1}{(2 n) !} \frac{\partial^{n}}{\partial t^{n}}(r-y(t)-b)^{2 n},
$$

where $y(t)$ is an infinitely differentiable function for $t>0$. It is clear that $w$ satisfies the following equations:

$$
\left\{\begin{array}{l}
\frac{\partial w}{\partial t}-\frac{\partial^{2} w}{\partial r^{2}}=0, \\
w(y(t)+b, t)=0, \\
2 b \dot{y}(t)=-\alpha \frac{\partial w}{\partial r}(y(t)+b, t) .
\end{array}\right.
$$

We take $y(t)=y_{\beta}(t) \equiv \beta \sqrt{t}$. Then, denoting the corresponding function (4. 9) by $w_{\beta}(r, t)$, we have

$$
w_{\beta}(b, t)=\frac{2 b}{\alpha} \sum_{n=1}^{\infty} \frac{n !}{(2 n) !} \beta^{2 n}
$$

and

$$
\frac{2 b}{\alpha}\left(\exp \left(\frac{\beta^{2}}{4}\right)-1\right)<w_{\beta}(b, t)<\frac{2 b}{\alpha}\left(\exp \left(\beta^{2}\right)-1\right) .
$$

Hence, putting $\beta_{1}=\left[\log \left(1+\frac{\alpha M}{2}\right)\right]^{1 / 2}$, we get

$$
w_{\beta_{1}}(b, t)<b M \quad\left(<w^{o}(b, t)\right) .
$$

Now we are ready to show the first inequality of (4.8) Suppose that it is false, then there would be a sufficiently small $t_{\tilde{\delta}}$ such that

$$
b+\beta_{1} \sqrt{t}<0^{o}(t) \quad\left(0 \leq t<t_{\hat{o}}\right), \quad b+\beta_{1} \sqrt{t}=\rho^{\delta}\left(t_{\delta}\right) .
$$


Then we have from (4.10) and (4.11), by the maximum principle,

$$
\tau_{\beta_{1}}(r, t)<w^{o}(r, t)
$$

for $0<r<b+\beta_{1} \sqrt{t}, 0<t<t_{\hat{0}}$. Hence, because of $w^{\delta}\left(\rho^{\delta}\left(t_{\delta}\right), t_{\hat{\delta}}\right)=w_{\beta_{1}}(b+$ $\left.\beta_{1} \sqrt{t_{\hat{o}}}, t_{\hat{o}}\right)=0$, we get by Friedman's lemma

$$
\frac{\partial w^{o}}{\partial r}\left(\rho^{\delta}\left(t_{\delta}\right), t_{\delta}\right)<\frac{\partial w_{\beta_{1}}}{\partial r}\left(\rho^{\delta}\left(t_{\delta}\right), t_{\delta}\right) .
$$

This means that

$$
\rho^{\delta}\left(t_{\delta}\right) \dot{\rho}^{\delta}\left(t_{\delta}\right)+\frac{\alpha m}{\rho^{\delta}\left(t_{\delta}\right)}>2 b \dot{y}\left(t_{\delta}\right)
$$

From (4.11) we have $\dot{\rho}^{\delta}\left(t_{\delta}\right) \leq \dot{y}\left(t_{\delta}\right)$, and therefore

$$
\frac{\alpha m}{b}>2 b \dot{y}\left(t_{\delta}\right)-\rho^{\delta}\left(t_{\delta}\right) \dot{\rho}^{\delta}\left(t_{\delta}\right)>b \dot{y}\left(t_{\delta}\right) .
$$

But this inequality contradicts to the fact that $\dot{y}\left(t_{\delta}\right)=\frac{\beta_{1}}{2 \sqrt{t_{\bar{\delta}}}}$ for sufficiently small $t_{\hat{\delta}}$. Thus we have proved that the first inequality of (4.8) holds for a small time interval $0<t<\varepsilon$.

Next we have from (4.3)

$$
\dot{\rho}^{\grave{o}}(t) \leq \frac{\alpha M}{\rho^{\hat{o}}(t)-b} \quad(t>0)
$$

and hence

$$
\frac{1}{2} \rho^{\delta}(t)^{2}-b \rho^{\delta}(t) \leq \frac{1}{2}(b+\delta)^{2}-b(b+\delta)+\alpha M t
$$

or

$$
\rho^{\delta}(t) \leq b+\sqrt{\delta^{2}+2 \alpha M t}<b+\delta<\beta_{2} \sqrt{t} \quad\left(\beta_{2}=\sqrt{2 \alpha M}\right) .
$$

Thus we have proved also the second inequality of (4.8).

It must be noted here for the later purpose that

$$
0<\dot{\rho}_{\delta}(t)<\frac{\alpha M}{\beta \sqrt{t}} \quad(0<t \leq \varepsilon)
$$

follows from (4.3) and (4.8).

Now we consider a sequence $\left\{\left(\rho^{\delta}, v^{\delta}\right)\right\}$ of solutions as $\delta \rightarrow 0$. Taking 
$\delta<1$ and putting $\sigma^{\hat{o}}(t) \equiv t\left(\rho^{\hat{o}}(t)-b\right)$, we have from (4.8)

$$
0 \leq \sigma^{\grave{o}}(t)<\varepsilon\left(1+\beta_{2} \sqrt{\varepsilon}\right) \quad(0<t \leq \varepsilon)
$$

and also from (4.8) and (4.12)

(4. 14) $0 \leq \dot{\sigma}^{\delta}(t)=\rho^{\delta}(t)-b+t \dot{\rho}^{\delta}(t) \leq 1+\left(\beta_{2}+\frac{\alpha M}{\beta_{1}}\right) \sqrt{\varepsilon} \quad(0 \leq t \leq \varepsilon)$.

Hence $\left\{\sigma^{\hat{o}}(t)\right\}$ are equicontinuous and uniformly bounded in $[0, \varepsilon]$. Therefore there is a subsequence which, denoted by $\left\{\sigma_{n}(t)\right\} \quad(n \rightarrow \infty)$, converges uniformly to a Lipschitz continuous function $\sigma(t)$. We put $\rho_{n}(t)=t^{-1} \sigma_{n}(t)$ and $\rho(t)=t^{-1} \sigma(t)$. Then we get $b+\beta_{1} \sqrt{t} \leq \rho(t) \leq b+$ $\beta_{2} \sqrt{t}(0 \leq t \leq \varepsilon)$ from (4.8) and $\rho_{n+1}(t)<\rho_{n}(t) \quad(t \geq 0)$ from (4.5).

We consider the solution $\left(\rho_{n}(t), v_{n}(r, t)\right)$ of $(4.1)$ and the solution $v(r, t)$ of the auxiliary problem

$$
\begin{cases}\frac{\partial v}{\partial t}-\Delta v=0 & (b<r<\rho(t), t>0), \\ v(b, t)=M & (t>0), \\ v(\rho(t), t)=\frac{m}{b}-\frac{m}{\rho(t)} & (t>0)\end{cases}
$$

with the prescribed boundary $\rho(t)$. From (4.3) and (4.8) we have

$$
\begin{gathered}
0 \leq v_{n}(\rho(t), t)-v_{n}\left(\rho_{n}(t), t\right) \leq \frac{M}{\beta_{1} \sqrt{t}}\left(\rho_{n}(t)-\rho(t)\right) \\
=\frac{M}{\beta_{1} t^{3 / 2}}\left(\sigma_{n}(t)-\sigma(t)\right) \quad(0<t \leq \varepsilon) .
\end{gathered}
$$

We know that for any $\eta>0$ there is an $n_{0}$ such that $\mid v_{n}\left(\rho_{n}(t), t\right)-$ $v(\rho(t), t) \mid<\eta$ for $n \geq n_{0}, 0 \leq t \leq \varepsilon$. Hence we get from (4.16) $v_{n}(\rho(t), t)$ $\rightarrow v(\rho(t), t) \quad(n \rightarrow \infty)$ uniformly for any time interval $t_{0} \leq t \leq \varepsilon$ being away from zero. By the maximum principle it follows that

$$
v_{n}(r, t) \rightarrow v(r, t) \quad(n \rightarrow \infty)
$$

uniformly for $0 \leq r \leq \rho(t), t_{0} \leq t \leq \varepsilon$. Consequently, since $t_{0}$ is arbitrary, we get

$$
v_{n}(r, t) \rightarrow v(r, t) \quad \text { for } 0 \leq r \leq \rho(t), 0<t \leq \varepsilon
$$

The resulting pair of functions $(\rho, v)$ turns out to be the desired 
solution of (3.5) in $0<t \leq \varepsilon$. In fact, by taking $n \rightarrow \infty \quad(\delta \rightarrow 0)$ along the extracted sequence in the formula (4.7), we have

$$
\begin{gathered}
\frac{1}{3 b}\left(\frac{1}{\alpha}-\frac{m}{b}\right)\left(\rho(t)^{3}-b^{3}\right)-\left(\frac{\alpha}{2}-\frac{m}{b}\right)\left(\rho(t)^{2}-b^{2}\right)-m(\rho(t)-b) \\
=m \int_{0}^{t} \frac{d \tau}{\rho(\tau)}-\int_{b}^{\rho(t)} r^{2}\left(\frac{1}{b}-\frac{1}{r}\right) v(r, t) d r+\left(M-\frac{m}{b}\right) t
\end{gathered}
$$

which is equivalent to the fourth condition of (3.5).

Next we get by (4.3) and (4.8) (in reality, those with $\delta=0$ )

$$
0 \leq v(r, \varepsilon)-\left(\frac{m}{b}-\frac{m}{\rho(\varepsilon)}\right) \leq \frac{M}{\beta_{1} \sqrt{\varepsilon}}(\rho(\varepsilon)-r)
$$

which corresponds to (2.9) in $\S 2$ that allows for the solution to exist also in $[\varepsilon, T]$. The proof is done as in $\S 2$.

Thus we have shown that there is a solution $(\rho, v)$ of (3.5) for any $(0, T)$. The uniqueness also can be shown in the same way as that in Theorem 2. Therefore we have

Theorem 3. There exists one and only one solution $(\rho, v)$ of the problem (3.5), and $\rho(t)$ satisfies the inequality

$$
\beta_{1} \sqrt{t} \leq \rho(t)-b \leq \beta_{2} \sqrt{t} \quad(t \geq 0)
$$

where $\beta_{1}$ and $\beta_{2}$ are positive constants.

\section{§5. Difference Scheme}

In the preceding sections we showed existence of solution of the problem (P). Here we will propose another method for solving the problem, that is, a finite difference method which is useful also for practical computation. We introduce a family of rectangular lattices on the $(r, t)$-plane with a uniform space mesh of width $h$ and time steps $k_{n}$ $(n=1,2, \cdots)$. We denote the discrete coordinates by

$$
r_{j}=\left(j-\frac{1}{2}\right) h \quad(j=0,1,2, \cdots)
$$

and 


$$
t_{n}=\sum_{p=1}^{n} k_{p} \quad(n=1,2,3, \cdots) .
$$

We choose $h$ so that $\left(b+\frac{1}{2} h\right) / h=J_{0}$ is an integer, while we find $k_{n}$ 's in the process of solving so that a free boundary (which is itself unknown, a priori) may cross each vertical line $r=r_{j}$ just on the corresponding discrete time $t=t_{n}$. We denote the free boundary points by $\left(y_{n}, t_{n}\right)$, where

$$
y_{n}=r_{J_{n}}=\left(J_{n}-\frac{1}{2}\right) h, \quad J_{n}=J_{0}+n \quad(n=1,2,3, \cdots) .
$$

With reference to given positive numbers $h$ and $k_{n}$ we denote $u\left(r_{j}, t_{n}\right)$ by $\iota_{j}^{n}$ occasionally and introduce the devided differences

$$
\begin{aligned}
& \left(u_{j}^{n}\right)_{r}=\left(u_{j+1}^{n}-u_{j}^{n}\right) / h, \\
& \left(u_{j}^{n}\right)_{\bar{r}}=\left(u_{j}^{n}-u_{j-1}^{n}\right) / h, \\
& \left(u_{j}^{n}\right)_{r \bar{r}}=\left(u_{j+1}^{n}-2 u_{j}^{n}+u_{j-1}^{n}\right) / h^{2}, \\
& \left(u_{j}^{n}\right)_{\bar{t}}=\left(u_{j}^{n}-u_{j}^{n-1}\right) / k_{n} .
\end{aligned}
$$

Our difference analogue to the problem (P) is the followings:

$$
\begin{cases}\Delta_{h} u_{j}^{n}=\left(u_{j}^{n}\right)_{i} & \left(0<j<J_{n}, n>0\right), \\ u_{J_{n}}^{n}=\frac{m}{b}-\frac{m}{y_{n}} & (n>0), \\ u_{1}^{n}=u_{0}^{n} & (n>0), \\ \frac{h}{k_{n}}=-\alpha v_{n}+\beta \frac{k_{n}}{\sqrt{h}} & (n>0 ; \beta \text { is a positive constant }), \\ u_{j}^{0}=f_{j} & \left(0 \leq j \leq J_{0}\right),\end{cases}
$$

where

$$
\lrcorner_{h} u_{j}^{n}=\left(u_{j}^{n}\right)_{r \bar{r}}+\frac{1}{r_{j}}\left(u_{j}^{n}\right)_{r}+\left(u_{j}^{n}\right)_{\bar{r}}, \quad v_{n}=\left(u_{J_{n}}^{n}\right)_{\bar{r}}
$$

The third equation of (5.1) corresponds to the situation of $\frac{\partial u}{\partial r}(0, t)$ $=0$ for the solution of the original problem $(\mathrm{P})$, which is to be automat cally satisfied because of spherical symmetry. The fourth equation of (5.1) is corresponding to $(\mathrm{P}-3)$ and contains an artificially added term $\beta k_{n} / \sqrt{h}$, which assures the convergence of our difference scheme as well 
as the convergence of an iteration procedure for solving the algebraic equations (5.1) for unknown $\left\{u_{j}^{n}\right\}$ and $k_{n}$. The quantities to be determined in our scheme are $\left\{k_{n}\right\} \quad(n=1,2, \cdots)$ and $\left\{u_{j}^{n}\right\} \quad\left(j=1,2, \cdots, J_{n}: n=1,2, \cdots\right)$, while those in the original problem $(\mathrm{P})$ are $y(l)(\iota>0)$ and $u(r, t)$ $(0<r<y(t), t>0)$.

In order to determine $k_{n}$ from the fourth equation of (5.1) we take the positive root:

$$
k_{n}=\frac{\sqrt{h}}{2 \beta}\left(\alpha v_{n}+\sqrt{\alpha^{2} v_{n}^{2}+4 \beta \sqrt{h}}\right), \quad \text { or }=-\frac{2 h}{\alpha v_{n}}-\frac{2}{\sqrt{\alpha^{2} v_{n}^{2}+4 \beta \sqrt{h}}} .
$$

An iteration procedure for finding $\left\{u_{j}^{n}\right\}$ and $k_{n}$ from (5.1) and (5.2) is to be described in $\$ 6$. In this section we consider the convergence of the scheme $(5.1)$ as $h \rightarrow 0$.

First of all, we prepare a maximum principle Suppose that $\left\{U_{j}^{n}\right\}$ satisfies

$$
\begin{cases}\Delta_{h} U_{j}^{n}-\left(U_{j}^{n}\right)_{i} \leq 0 & \left(0<j<J_{n}, n>0\right) \\ U_{J_{n}}^{n}=F_{n}(\geq 0) & (n \geq 0) \\ U_{1}^{n} \leq U_{0}^{n} & (n \geq 0) \\ U_{i}^{\prime \prime}=f_{j}(\geq 0) & \left(0<j \leq J_{0}\right)\end{cases}
$$

Then we have

Lemma 6. $U_{j}^{n} \geq 0$ holds for $0 \leq j \leq J_{n}$ and $n>0$. If, further, $f_{j}$ $\neq 0$, the strict inequality holds: $U_{j}^{n}>0$.

Proof. By putting $r_{j} U_{j}^{n}=V_{j}^{n}$ we can reduce (5.3) to the following:

$$
\begin{cases}\left(V_{j}^{n}\right)_{r \bar{r}}-\left(V_{j}^{n}\right)_{\bar{t}} \leq 0 & \left(0<j<J_{n}, n>0\right), \\ V_{J_{n}}^{n}=r_{J_{n}} F_{n}(\geq 0) & (n>0), \\ V_{1}^{n}+V_{0}^{n} \leq 0 & (n>0) \\ V_{j}^{0}=r_{j} f_{j} & \left(0 \leq j \leq J_{0}\right) .\end{cases}
$$

If there were a point $\left(r_{j_{0}}, t_{n_{0}}\right) \quad\left(j_{0}>0\right)$ such that

$$
\begin{gathered}
V_{j}^{n} \geq 0 \text { for } 0<j \leq J_{n}, 0 \leq n \leq n_{n}-1 ; \quad V_{j_{0}}^{n_{0}}<0 ; \\
V_{j}^{n_{0}} \geq V_{j_{0}}^{n_{0}} \text { for } j \neq j_{0}, j \geq 1,
\end{gathered}
$$


then it should follow that

$$
\left(V_{j_{0}}^{n_{0}}\right)_{r \bar{r}} \geq 0 \quad \text { and } \quad\left(V_{j_{0}}^{n_{0}}\right)_{\bar{t}}<0
$$

This is, however, a contradiction to the first equation of (5.4). Hence we get $V_{j}^{n} \geq 0$ and also $U_{j}^{n} \geq 0$ for $1 \leq j \leq J_{n}, n \geq 0$, and $U_{0}^{n}\left(\geq U_{1}^{n}\right) \geq 0$.

Next we suppose that $f(r) \geq 0$ and $f(r) \not \equiv 0$. If there were a point $\left(r_{j_{1}}, t_{1}\right) \quad\left(j_{1} \geq 1\right)$ such that $V_{j_{1}}^{1}=0$, then $\left(V_{j_{1}}^{1}\right)_{r \bar{r}} \leq 0$ hold from the first equation of (5.4), and hence $V_{j_{1} \pm 1}^{1}=0$ from the fact of $V_{j}^{n} \geq 0$ proven just above. Repeating this argument yields $V_{j}^{1}=0$ for all $1 \leq j \leq J_{1}$. However, at a point $r_{j_{2}}$ such that $f_{j_{2}}>0$, we have $\left(V_{j_{2}}^{1}\right)_{\bar{t}}<0$ and $\left(V_{j_{2}}^{1}\right)_{r \bar{r}}$ $=0$. But this contradicts to the first equation of (5.4). Hence we get $V_{j}^{1}>0$ for $1 \leq j \leq J_{1}$. By continuing the argument successively we have $V_{j}^{n}>0$ and also $U_{j}^{n}>0$ for $1 \leq j \leq J_{n}, n>0$, and hence also $U_{0}^{n}>0$.

Q.E.D.

The next lemma is essential for the proof of the convergence of our difference scheme. It assures that $\left\{k_{n}\right\} \quad(n>0)$ tend to zero uniformly as $h \rightarrow 0$.

Lemma 7. There are positive constants $c_{i}(i=1,2)$ such that

$$
v_{n} \equiv\left(u_{J_{n}}^{n}\right)_{\bar{r}} \leq c_{1} h^{3 / 8} \quad(n>0)
$$

and

$$
k_{n} \leq c_{2} h^{3 / 4} \quad(n>0)
$$

Proof. First of all we consider the case that all $\left\{\boldsymbol{v}_{n}\right\}$ are nonpositive. Then (5.5) is trivial and $k_{n} \leq h^{3 / 4} / \sqrt{\beta}$ follows from (5.2). Thus (5. 6) holds with a constant $c_{2}>1 / \sqrt{ } \bar{\beta}$.

Next we consider the case that some of $\left\{v_{n}\right\} \quad(n=1,2, \cdots)$ may be positive. Put

$$
\left(u_{j}^{n}\right)_{\bar{r}}=z_{j}^{n} \quad\left(1 \leq j \leq J_{n}, n>0\right)
$$

Then $\left\{z_{j}^{n}\right\}$ satisfy the following equations: 


$$
\begin{cases}\left(z_{j}^{n}\right)_{r \bar{r}}+\frac{1}{r_{j}}\left\{\left(z_{j}^{n}\right)_{r}+\left(z_{j}^{n}\right)_{\bar{r}}\right\}-\frac{1}{r_{j} r_{j-1}}\left(z_{j}^{n}+z_{j-1}^{n}\right)=\left(z_{j}^{n}\right)_{\bar{t}} \\ & \left(2 \leq j \leq J_{n}-1, n>0\right) \\ z_{1}^{n}=0 & (n>0) \\ z_{j}^{0}=\left(f_{j}\right)_{\vec{r}} & \left(1 \leq j \leq J_{0}\right) .\end{cases}
$$

And $\left\{z_{j}^{n}\right\} \quad\left(1 \leq j \leq J_{n}, 1 \leq n \leq N\right)$ take their positive maximum just on the boundary $\left(x_{J_{n}}, t_{n}\right) \quad(1 \leq n \leq N)$. Otherwise the positive maximum would occur at an internal point $\left(r_{j_{0}}, t_{n_{0}}\right) \quad\left(1 \leq j_{0} \leq J_{n_{0}}-1\right)$. Clearly $j_{0} \neq 1$ since $z_{1}{ }^{n}=0$. The difference equation to be satisfied at $\left(r_{j_{0}}, t_{n_{0}}\right)$ can be described explicitly as

$$
\begin{gathered}
\left(\frac{1}{h^{2}}+\frac{1}{h r_{j_{0}}}\right)\left(z_{j_{0}+1}^{n_{0}}-z_{j_{0}}^{n_{0}}\right)-\left(\frac{1}{h^{2}}+\frac{1}{h r_{j_{0}}}+\frac{1}{r_{j_{0}} r_{j_{0}-1}}\right) z_{j_{0}}^{n_{0}} \\
+\left(\frac{1}{h^{2}}-\frac{1}{h r_{j_{0}}}-\frac{1}{r_{j_{0}} r_{j_{0}-1}}\right) z_{j_{0}-1}^{n_{0}}=\left(z_{j_{0}}^{n_{0}}\right)_{i} .
\end{gathered}
$$

From the assumption, we have

$$
\begin{aligned}
& \left(\frac{1}{h^{2}}+\frac{1}{h r_{j_{0}}}\right)\left(z_{j_{0}+1}^{n_{0}}-z_{j_{0}}^{n_{0}}\right) \leq 0, \\
& -\left(\frac{1}{h^{2}}-\frac{1}{h r_{j_{0}}}+\frac{1}{r_{j_{0}} r_{j_{0}-1}}\right) z_{j_{0}}^{n_{0}} \leq-\frac{5}{3 h^{2}} z_{j_{0}}^{n_{0}}<0 .
\end{aligned}
$$

For $j_{0}=2$, we have $z_{j_{0}-1}^{n_{0}}=0$, and for $j_{0} \geq 3$,

$$
\frac{1}{h^{2}}-\frac{1}{h r_{j_{0}}}-\frac{1}{r_{j_{0}} r_{j_{0}-1}} \geq \frac{1}{3 h^{2}} \text {. }
$$

In both cases, we get

$$
-\left(\frac{1}{h^{2}}-\frac{1}{h r_{j_{0}}}-\frac{1}{r_{j_{0}} r_{j_{0}-1}}\right) z_{j_{0}}^{n_{0}} \leq 0 .
$$

From these inequalities we find that the left hand side of (5.7) becomes negative, while the right hand side is non-negative from our assumption. This is a contradiction. Therefore $\left\{z_{j}^{n}\right\}$, if some of them become positive, should take their maximum at a point $\left(r_{J_{0}}, t_{n_{0}}\right)$ on the free boundary.

Put

$$
u_{j}^{n}-\left(\frac{m}{b}-\frac{m}{y_{n}}\right)=U_{j}^{n}
$$


We find that $\left\{U_{j}^{n}\right\}$ satisfy

$$
\begin{cases}\Delta_{h} U_{j}^{n}-\left(U_{j}^{n}\right)_{\bar{t}}=\frac{h}{y_{n} y_{n-1}} & \left(1 \leq j \leq J_{n}-1, n>0\right) \\ U_{J_{n}}^{n}=0 & (n>0)\end{cases}
$$

Since $z_{J_{n_{0}}}^{n_{0}}=\left(u_{J_{n_{0}}-1}^{n_{0}}\right)_{r} \geq\left(u_{J_{n_{0}}-1}^{n_{0}}\right)_{\bar{r}}$ and $\frac{1}{h}-\frac{1}{r_{J_{n_{0}}-1}}>0$ for sufficiently small $h$, we have

$$
\Delta_{h} U_{J_{n_{0}}-1}^{n_{0}}=\Delta_{h} u_{J_{n_{0}}-1}^{n_{0}}=\left(\frac{1}{h}+\frac{1}{r_{J_{n_{0}}-1}}\right)\left(u_{J_{n_{0}}-1}^{n_{0}}\right)_{r}-\left(\frac{1}{h}-\frac{1}{r_{J_{n_{0}}-1}}\right)\left(u_{J_{n_{0}}-1}^{n_{0}}\right)_{\bar{r}}>0 .
$$

Since $U_{J_{n_{0}-1}}^{n_{0}}<0$ and $U_{J_{n_{0}}-1}^{n_{0}-1}=0$, we get $\left(U_{J_{n_{0}}-1}^{n_{0}}\right)_{\bar{t}}<0$. Hence we get from (5.8)

$$
0<-\left(U_{J_{n_{0}}-1}^{n_{0}}\right)_{\bar{t}} \leq \frac{h}{b^{2}}
$$

because $y_{n_{0}}$ and $y_{n_{0}-1}>b$. Therefore by $U_{J_{n_{0}}}^{n_{0}}=U_{J_{n_{0}}-1}^{n_{0}-1}=0$, we have

$$
\left(U_{J_{n_{0}}}^{n_{0}}\right)_{\bar{r}}=-\frac{k_{n_{0}}}{h}\left(U_{J_{n_{0}-1}}^{n_{0}}\right)_{\bar{t}} \leq \frac{k_{n_{0}}}{b^{2}}
$$

Thus we find

$$
v_{n_{0}}=\left(u_{J_{n_{0}}}^{n_{0}}\right)_{\bar{r}} \leq \frac{k_{n_{0}}}{b^{2}}
$$

or, by (5.2),

$$
\stackrel{h}{\frac{h}{k_{n_{0}}}} v_{n_{0}}=\frac{2 \beta \sqrt{h} v_{n_{0}}}{\sqrt{\alpha^{2} v_{n_{0}}^{2}+4 \beta \sqrt{h}}+\alpha v_{n_{0}}} \leq \frac{h}{b^{2}}
$$

Hence we have $v_{n_{0}} \leq \frac{h^{3 / 8}}{\alpha \sqrt{\beta b^{2}}}$. Since $n_{0}$ is arbitrary, we get finally

$$
v_{n} \leq \frac{h^{3 / 8}}{\alpha \sqrt{\beta} b^{2}} \text { for any } n>0
$$

and, from (5.2),

$$
k_{n} \leq c_{2} h^{3 / 1} . \quad \text { Q.E.D. }
$$

Lemma 8. We take the same constant $N$ as in (2.9). The following inequalities hold: 


$$
-v_{n} \leq \frac{N}{y_{n-1} y_{n}}<\frac{N}{b^{2}} \quad(n>0)
$$

$$
\frac{h}{k_{n}} \leq \frac{\alpha N}{b^{2}}+1 \quad\left(\equiv N_{1}\right) \quad(n>0)
$$

and

$$
\left(u_{J_{n}-1}^{n}\right)_{\bar{t}}<\frac{1}{b^{2}}\left(\frac{\alpha N}{b^{2}}+1\right)(N+m) \quad(n>0) .
$$

Proof. For comparison, we take a 'barrier' function

$$
\omega_{n_{0}}\left(r_{j}, t_{n}\right)=N\left[\frac{1}{\left|r_{j}\right|}-\frac{1}{y_{n_{0}}}\right]+\frac{m}{b}-\frac{m}{y_{n_{0}}} .
$$

It can be easily seen that

$$
\begin{array}{cc}
\lrcorner_{h}\left\{\omega_{n_{0}}\left(r_{j}, t_{n}\right)\right\}-\left\{\omega_{n_{0}}\left(r_{j}, t_{n}\right)\right\}_{\bar{t}}=0 & \left(1 \leq j<J_{n}, 0<n \leq n_{0}\right), \\
\Delta_{h}\left\{\omega_{n_{0}}\left(r_{1}, t_{n}\right)\right\}-\left\{\omega_{n_{0}}\left(r_{1}, t_{n}\right)\right\}_{\bar{t}}=-\frac{4 N}{h^{3}}<0 & \left(0<n \leq n_{0}\right), \\
\omega_{n_{0}}\left(y_{n}, t_{n}\right)=N\left[\frac{1}{y_{n}}-\frac{1}{y_{n_{0}}}\right]+\frac{m}{b}-\frac{m}{y_{n_{0}}} \geq \frac{m}{b}-\frac{m}{y_{n_{0}}} & \left(0 \leq n \leq n_{0}\right), \\
\left(\omega_{n_{0}}\left(r_{1}, t_{n}\right)=\omega_{n_{0}}\left(r_{0}, t_{n}\right)\right. & \left(0<n \leq n_{0}\right), \\
\omega_{n_{0}}\left(r_{j}, 0\right)=N\left[\frac{1}{r_{j}}-\frac{1}{y_{n_{0}}}\right]+\frac{m}{b}-\frac{m}{y_{n_{0}}} & \\
>N\left[\frac{1}{r_{j}}-\frac{1}{y_{n_{0}}}\right]>N\left[\frac{1}{r_{j}}-\frac{1}{b}\right] \geq f\left(r_{j}\right) & \left(1 \leq j \leq J_{0}\right) .
\end{array}
$$

Therefore it follows from Lemma 6 that

$$
\left(\omega_{n_{0}}\left(r_{j}, t_{n}\right) \geq u_{j}^{n} \quad\left(0 \leq j \leq J_{n}, n>0\right),\right.
$$

and hence

$$
\frac{\left\{u_{J_{n_{0}}-1}^{n_{0}}-\left(\frac{m}{b}-\frac{m}{y_{n_{0}}}\right)\right\}}{h} \leq \frac{N}{\left(y_{n_{0}}-h\right) y_{n_{0}}}
$$

which yields

$$
-v_{n_{0}} \leq \frac{N}{y_{n_{0}-1} y_{n_{0}}}<\frac{N}{b^{2}}
$$

Since $n_{0}$ is arbitrary, we get (5.9). From (5.9) and (5.2) we get also (5. 10): for small $h$, 


$$
\frac{h}{k_{n}}=-\frac{1}{2}\left(\alpha v_{n}-\sqrt{\alpha^{2} v_{n}^{2}+4 \beta \sqrt{h}}\right)<\frac{\alpha N}{b^{2}}+1 .
$$

The function

$$
U_{j}^{n} \equiv u_{j}^{n}-\left(\frac{m}{b}-\frac{m}{y_{n}}\right)
$$

satisfies the inequality

$$
\left(U_{J_{n}-1}^{n}\right)_{\bar{t}}=-\frac{h}{k_{n}}\left(U_{J_{n}}^{n}\right)_{\bar{r}}=-\frac{h}{k_{n}} v_{n} \leq \frac{N}{b^{2}}\left(\frac{\alpha N}{b^{2}}+1\right)
$$

since $U_{J_{n}}^{n}=U_{J_{n}-1}^{n-1}=0$. Hence

$$
\begin{aligned}
\left(u_{J_{n}-1}^{n}\right)_{\bar{t}} & =\left(U_{J_{n}-1}^{n}\right)_{\bar{t}}+\frac{m}{k_{n}}\left(\frac{1}{y_{n-1}}-\frac{1}{y_{n}}\right) \\
& <\frac{1}{b^{2}}\left(\frac{\alpha N}{b^{2}}+1\right)(N+m) .
\end{aligned}
$$

Thus we have proved Lemma 8.

Next we assume that, in addition to (2.1) and (2.8), $f^{\prime}(r)$ and $\Delta f(r)$ exist and there are positive constants $M_{i}(i=1,2)$ such that

$$
\left|f^{\prime}(r)\right| \leq M_{1} \text { and }|\Delta f(r)| \leq M_{2} \text { for } 0 \leq r \leq b .
$$

Lemma 9. Under the above assumption the solution $\left\{u_{j}^{u}\right\}$ have the estimates

$$
\left|\left(u_{j}^{n}\right)_{\bar{r}}\right| \leq M_{1} \text { and }\left|\left(u_{j}^{n}\right)_{\bar{t}}\right| \leq M_{2} \text { for } 0<j \leq J_{n}, n>0 .
$$

Proof. We first note that $f(r)$ satisfies (2.9) with the constant $N=b^{2} M_{1}$ (if it is necessary, we take $M_{1}$ sufficiently large).

The function

$$
W_{j}^{n}=M_{1} \pm\left(u_{j}^{n}\right)_{\bar{r}}
$$

satisfies the following equations:

$$
\begin{cases}\Delta_{h} W_{j}^{n}-\left(W_{j}^{n}\right)_{i}=0 & \left(1<j<J_{n}, n>0\right), \\ W_{J_{n}}^{n}=M_{1} \pm\left(u_{J_{n}}^{n}\right)_{\bar{r}} \geq 0 & (n>0), \\ W_{1}^{n}=M_{1}>0 & (n>0), \\ W_{j}^{0}=M_{1} \pm\left(f_{j}\right)_{\vec{r}} \geq 0 . & \end{cases}
$$


By the same argument as in the proof of Lemma 6 we get $W_{j}^{n} \geq 0$, that is, $\left|\left(u_{j}^{n}\right)_{\bar{r}}\right| \leq M_{1}$.

Next the function

$$
Z_{j}^{n}=M_{2} \pm\left(u_{j}^{n}\right)_{\bar{\imath}}
$$

(where we take $M_{2}$ larger than $\frac{1}{b^{2}}\left(\frac{N}{b^{2}}+1\right)(N+m)$ ) satisfies the following equations:

$$
\begin{cases}\Delta_{n} Z_{j}^{n}-\left(Z_{j}^{n}\right)_{i}=0 & \left(0<j<J_{n}-1, n>0\right) \\ Z_{J_{n}-1}^{n}=M_{2} \pm\left(u_{J_{n}-1}^{n}\right)_{i} \geq 0 & (n>0) \\ Z_{1}^{n}=Z_{0}^{n} & \\ Z_{j}^{0}=M_{2} \pm \Delta_{h} f\left(r_{j}\right) \geq 0 . & \end{cases}
$$

By applying Lemma 6 we get $Z_{j}^{n} \geq 0$, that is, $\left|\left(u_{j}^{n}\right)_{i}\right| \leq M_{2}$. Thus we have proved Lemma 9.

Now we will go to the proof of convergence of our difference scheme under the assumptions (2.1), (2.8) and (5.12). First we have to notice that $\left\{k_{n}\right\}$ tend to zero uniformly as $h \rightarrow 0$ by Lemma 7 . We define the piecewise linear and continuous function $y_{n}(t)$ as follows:

$$
\begin{array}{r}
y_{h}(t)=\frac{t-t_{n}}{k_{n}} y_{n+1}(t)+\frac{t_{n+1}-t}{k_{n}} y_{n}(t) \quad \text { for } t_{n} \leq t \leq t_{n+1} \\
(n=0,1,2, \cdots) .
\end{array}
$$

Then, by (5.10) of Lemma 8, $y_{h}(t)$ is Lipschitz continuous with the uniform Lipschitz constant $N_{1}$ and uniformly bounded for any closed interval $0 \leq t \leq T$. Hence, by the Ascoli-Arzela's theorem, there is a subsequence of $\left\{y_{h}(t)\right\}$ (which we denote again by $\left\{y_{h}(t)\right\}$ ) such that $y_{h}(t)$ converges uniformly to a Lipschitz continuous function $y(t)$ as $h$ tends to zero through the selected sequence.

It follows from Lemma 9 that $\left\{\left|\left(u_{j}^{n}\right)_{r \bar{r}}\right|\right\}$ are uniformly bounded for $\varepsilon<r_{j}<y_{n}, 0<l_{n} \leq T$ where $\varepsilon$ is any small positive constant. It also can be seen that $\left\{u_{r \bar{r} r}\right\},\left\{u_{r \bar{r} t}\right\}$ and $\left\{u_{\bar{r} r}\right\}$ are uniformly bounded for $\varepsilon<r_{j}<$ $y(t)-\varepsilon, \varepsilon<t_{n} \leq T$. Indeed, if we put $(j-1 / 2) h u_{j}^{n}=r v_{j}^{n}$, we get the equation to be satisfied by $w_{j}^{n}$ 


$$
\left(w_{j}^{n}\right)_{r \bar{r}}-\left(w_{j}^{n}\right)_{\bar{t}}=0,
$$

and therefore we find that $\left\{w_{j}^{n}\right\}$ are uniformly bounded for $0<r_{j} \leq y_{n}$, $0 \leq t_{n} \leq T$. Then any divided differences of higher order of $\left\{w_{j}^{n}\right\}$ with respect to both $t$ and $r$ are, as well known, uniformly bounded for any fixed closed region with a finite distance from the boundary of the region $\{0<r<y(t), 0<t<T\}$ (see Petrowsky's text book [10]). Hence any divided differences of $\left\{u_{j}^{n}\right\}$ are also uniformly bounded in such closed region. From this one can see that there is a subsequence $\left\{u_{j}^{n}\right\}$ (where we use the same notation for the subsequence as the original one) which converges to an infinitely differentiable function $u(r, t)$ in $\{0<r<y(t)$, $0<t<T\}$. (In reality we must take the sequence of the extended functions from that of the grid functions $\left\{u u_{j}^{n}\right\}$ over the whole region $\{0<r<$ $y(t), 0<t<T\}$ by a definite method, but we omit the discussion here and later.) The divided differences $\left\{\left(u_{j}^{n}\right)_{r \bar{r}}\right\},\left\{\left(u_{j}^{n}\right)_{\bar{t}}\right\}$ and $\left\{\left(u_{j}^{n}\right)_{r}\right\}$ also converge to the corresponding derivatives $\frac{\partial^{2} u}{\partial r^{2}}, \frac{\partial u}{\partial t}$ and $\frac{\partial u}{\partial r}$ respectively. The convergences are uniform in any closed region in $\{0<r<y(t)$, $0<t<T\}$.

Tending $h$ to zero through the extracted subsequence, we obtain from the first equation of (5.1)

$$
\Delta u=\frac{\partial u}{\partial t} \quad(0<r<y(t), 0<t \leq T) .
$$

Since $\left(u_{j}^{n}\right)_{\bar{t}}$ are uniformly bounded in the whole region $\{0<r<y(t), 0<t$ $\leq T\}$ by Lemma 9 , limit functions $\frac{\partial u}{\partial t}$ and $\Delta u$ also are bounded there. One can see from this that $\lim _{t \rightarrow 0} u(r, t)=u(r,+0)$ exists and is equal to $f(r)$. Similarly we find that $\frac{\partial u}{\partial r}$ is also bounded in the whole region, and hence that $\lim _{r \rightarrow 0} u(r, t)=u(+0, t)$ and $\lim _{r \rightarrow y(t)-0} u(r, t)=u(y(t)-0, t)$ exist and the latter is equal to $\frac{m}{b}-\frac{m}{y(t)}$. This means that $u(r, t)$ is continuous in the closed region $0 \leq r \leq y^{\prime}(t), 0 \leq t \leq T$. Finally, since $\left\{\left(u_{j}^{n}\right)_{r \bar{r}}\right\}$ are uniformly bounded in $\{\varepsilon<r<y(t), 0<t \leq T\}$ (for any $\varepsilon>0$ ), $\frac{\partial^{2} u}{\partial r^{2}}$ is bounded in the same region. Hence $\lim _{r \rightarrow y(t)-0} \frac{\partial u}{\partial r}(r, t)=v(t)$ exists, where the convergence is uniform in $0<t \leq T$, and $v(t)$ is continuous in $0<t \leq T$.

The fourth equation of (5.1) yields 


$$
y_{n}=b-\alpha \sum_{p=1}^{n} k_{p} v_{p}+\sum_{p=1}^{n} \beta \frac{k_{p}^{2}}{\sqrt{h}} .
$$

Tending $h$ to zero and supposing that $\sum_{p=1}^{n} k_{p}$ tend to $t$, we find by Lemma 7 that the last term of the above relation tends to zero, and that

$$
y(t)=b-\alpha \int_{0}^{t} v(\tau) d \tau
$$

holds as the limit. Hence $y(t)$ is differentiable and

$$
\dot{v}(t)=-\alpha v(t) .
$$

Thus we have shown that the pair of the limit functions $(y, u)$ satisfies all the conditions of the system (P), and therefore it is a solution of (P). We can see from the uniqueness theorem (Theorem 1) that all the sequence $\left\{y_{n}, u_{j}^{n}\right\}$ converge to the solution $(y, u)$. The proof of convergence is completed. Thus we have

Theorem 4. Under the assumption of (2.1), (2.8) and (5.12), the solution $\left(y_{n}, u_{j}^{n}\right)$ of our difference scheme (5.1) converges to the solution $(y, u)$ of the original problem (P) as $h \rightarrow 0$.

Remark 1. The above proof of convergence is another proof of the existence theorem of the solution of $(\mathrm{P})$ at the same time. While the imposed assumption is stronger than that of Theorem 2, the extra ones could be removed by some approximating technique. (To remove (2.8) was already performed in $\$ 3$.)

Remark 2. In our scheme (5.1) we introduced the artificial term $\beta k_{n} / \sqrt{h}$. We have shown that the convergence is obtained whenever $\beta$ is set any positive value. In pratical computation, however, the space mesh $h$ is set as a finitely small constant, and hence $\beta$ must be taken so small that the artificial term may not exceed the principal terms. Otherwise the accuracy of solution would go down. On the other hand, as we shall see in the following section, $\beta$ must be taken so large independently of $h$ that the iteration procedure of finding $\left\{u_{j}^{n}\right\}$ and $k_{n}$ from the algebraic equations (5.1) on each time step $n$ may converge. Hence 
we may determine the value of $\beta$ as follows: once $h$ is fixed, by performing test computations with several $\beta$-values in decreasing order, we choose the least one among them that may admit the convergence of iteration and by using it we perform the full computation. We will give one more brief comment for practical computation. While the convergence was proved above in the case of $\beta$ fixed, one may use variable $\beta$ 's for practical computation and especially may put some $\beta$ 's $=0$ as far as the iterations converge.

\section{§ 6. Iteration Method for Solving our Difference Scheme}

In order to get $k_{n}$ and $\left\{u_{j}^{n}\right\}$ from the nonlinear algebraic equation (5.1) at each time step $t=t_{n}$, we will use the following iteration method:

$$
\left\{\begin{array}{l}
k_{n}^{(0)}=k_{n-1} \quad\left(k_{0}=\text { an arbitrary positive constant }\right) \\
u_{r \bar{r}}^{(s)}\left(r_{j}, t_{n}\right)+\frac{1}{r_{j}}\left(u_{r}^{(s)}\left(r_{j}, t_{n}\right)+u_{\bar{r}}^{(s)}\left(r_{j}, t_{n}\right)\right) \\
\quad-\frac{u^{(s)}\left(r_{j}, t_{n}\right)-u\left(r_{j}, t_{n-1}\right)}{k_{n}^{(s)}}=0 \\
u^{(s)}\left(r_{1}, t_{n}\right)=u^{(s)}\left(r_{0}, t_{n}\right), \\
u^{(s)}\left(r_{J_{n}}, t_{n}\right)=\frac{m}{b}-\frac{m}{y_{n}}, \quad(s=0,1,2, \cdots) \\
k_{n}^{(s+1)}=\frac{\sqrt{h}}{2 \beta}\left(\alpha v_{n}^{(s)}+\sqrt{\alpha^{2} v_{n}^{(s) 2}+4 \beta \sqrt{h}}\right)
\end{array}\right.
$$

where $v_{n}^{(s)}=u u_{\bar{r}}^{(s)}\left(r_{J_{n}}, t_{n}\right)$.

Theorem 5. For any sufficiently large positive constant $\beta$ the above iteration procedures converge uniformly with respect to $n$ as $s \rightarrow \infty$ and the limits $k_{n}^{(\infty)}$ and $u^{(\infty)}\left(r_{j}, t_{n}\right)$ are the solution of $(5.1)$.

Proof. Put

$$
r_{j} u^{(s)}\left(r_{j}, t_{n}\right)=w^{(s)}\left(r_{j}, t_{n}\right) .
$$

Then we get easily the following system to be satisfied by $w^{(s)}$ and $k_{n}^{(s)}$, 
(6. 3)

$$
\left\{\begin{array}{l}
k_{n}^{(0)}=k_{n-1}, \\
w_{r \bar{r}}^{(s)}\left(r_{j}, t_{n}\right)-\frac{w^{(s)}\left(r_{j}, t_{n}\right)-w\left(r_{j}, t_{n}\right)}{k_{n}^{(s)}}=0, \\
w^{(s)}\left(r_{J+n}, t_{n}\right)+w^{(s)}\left(r_{0}, t_{n}\right)=0, \\
w^{(s)}\left(r_{J+n}, t_{n}\right)=y_{n}\left(\frac{m}{b}-\frac{m}{y_{n}}\right) .
\end{array}\right.
$$

We introduce one-step-Green's functions related to the half-plane problems of the pure implicit difference analogue $w_{r \bar{r}}-w_{\bar{t}}=0$ of the heat equation $\frac{\partial^{2} w}{\partial r^{2}}-\frac{\partial w}{\partial t}=0$,

(6. 4) $g\left(r_{j}, \xi_{l} ; t_{n}\right)=\frac{1}{2 \pi h} \int_{-\pi}^{\pi}\left(1+4 \lambda_{n} \sin ^{2} \frac{\omega}{2}\right)^{-1}$

$$
\begin{gathered}
\times\left[\exp \left\{-i\left(r_{j}-\xi_{l}\right) \frac{\omega}{h}\right\}-\exp \left\{-i\left(r_{j}+\xi_{l}\right) \frac{\omega}{h}\right\}\right] d(1) \\
G\left(r_{j}, \xi_{l} ; t_{n}\right)=\frac{1}{2 \pi h} \int_{-\pi}^{\pi}\left(1+4 \lambda_{n} \sin ^{2} \frac{\omega}{2}\right)^{-1} \\
\times\left[\exp \left\{-i\left(r_{j}-\xi_{l}\right) \frac{\omega}{h}\right\}+\exp \left\{-i\left(r_{j}+\xi_{l}-1\right) \frac{\omega}{h}\right\}\right] d \omega,
\end{gathered}
$$

where $\lambda_{n}=\frac{k_{n}}{h^{2}}$ and $\xi_{l}=\left(l-\frac{1}{2}\right) h$ as well as $r_{j}=\left(j-\frac{1}{2}\right) h$. One can easily see that the equation

$$
g_{\bar{\xi} \bar{\xi}}\left(r_{j}, \xi_{l} ; t_{n}\right)-\frac{g\left(r_{j}, \xi_{l} ; t_{n}\right)-\delta_{j, l}}{k_{n}}=0\left(\delta_{j, l}=\left\{\begin{array}{l}
\frac{1}{h}(j=l) \\
0(j \neq l)
\end{array}\right)\right.
$$

and the boundary condition

$$
g\left(r_{j}, \tilde{\xi}_{1} ; t_{n}\right)+g\left(r_{j}, \hat{亏}_{0} ; t_{n}\right)=0
$$

and the conjugate relations

$$
g_{\bar{r}}=-G_{\hat{\xi}}
$$

are satisfied.

By using the Green's function $g$ and the property (6.6) we can give the following representation of $w^{(s)}\left(r_{j}, t_{n}\right)$

$$
w^{(s)}\left(r_{j}, t_{n}^{(s)}\right)=\sum_{i=1}^{s_{n}-1} h g\left(r_{j}, \xi_{l} ; t_{n}^{(s)}\right) w\left(\xi_{l}, t_{n-1}\right)
$$




$$
\begin{aligned}
& +k_{n}^{(s)} g\left(r_{j}, y_{n} ; t_{n}^{(s)}\right) w_{\bar{r}}^{(s)}\left(y_{n}, t_{n}^{(s)}\right) \\
& -k_{n}^{(s)} g_{\bar{\xi}}\left(r_{j}, y_{n} ; t_{n}^{(s)}\right) w_{n}^{(s)}\left(y_{n}, t_{n}\right)
\end{aligned}
$$

where $t_{n}^{(s)}=t_{n-1}+k_{n}^{(s)}$. (Refer to [9].) Hence, by (6.7) we get

$$
\begin{aligned}
w_{\bar{r}}^{(s)}\left(y_{n}, t_{n}\right)= & -\sum_{i=1}^{J_{n}-1} h G_{\xi}\left(y_{n}, \xi_{l} ; t_{n}^{(s)}\right) w\left(\xi_{l}, t_{n-1}\right) \\
& -k_{n}^{(s)} G_{\xi}\left(y_{n}, y_{n} ; t_{n}^{(s)}\right) w_{\bar{r}}^{(s)}\left(y_{n}, t_{n}^{(s)}\right) \\
& +k_{n}^{(s)} G_{\xi \bar{\xi}}\left(y_{n}, y_{n} ; t_{n}^{(s)}\right) w^{(s)}\left(y_{n}, t_{n}^{(s)}\right)
\end{aligned}
$$

or

$$
\begin{aligned}
w_{\bar{r}}^{(s)}\left(y_{n}, t_{n}\right)= & {\left[1+k_{n}^{(s)} G_{\xi}\left(y_{n}, y_{n} ; t_{n}^{(s)}\right)\right]^{-1} } \\
& \times\left[k_{n}^{(s)} G_{\xi \bar{\xi}}\left(y_{n}, y_{n} ; t_{n}^{(s)}\right) w^{(s)}\left(y_{n}, t_{n}^{(s)}\right)\right. \\
& \left.-\sum_{l=1}^{J_{n}-1} h G_{\xi}\left(y_{n}, \xi_{l} ; t_{n}^{(s)}\right) w\left(\xi_{l}, t_{n-1}\right)\right] .
\end{aligned}
$$

Hence we have by the same way as in $\S 3$ of [9]

$$
\left|w_{\bar{r}}^{(s)}\left(y_{n}, t_{n}\right)-w_{\bar{r}}^{(s-1)}\left(y_{n}, t_{n}\right)\right|<\frac{\text { const. }}{\beta}\left|w_{\bar{r}}^{(s-1)}\left(y_{n}, t_{n}\right)-w_{\bar{r}}^{(s-2)}\left(y_{n}, t_{n}\right)\right|,
$$

where the constant on the right hand side does not depend on any of $s, n$ and $h$. It follows from the last relations that $\left\{w_{r}^{(s)}\left(y_{n}, t_{n}\right)\right\}$ converge to a limit $w_{\bar{\tau}}\left(y_{n}, t_{n}\right)$ as $s \rightarrow \infty$ for a sufficiently large constant $\beta$. Then $\left\{v_{n}^{(s)}\right\}$ and $\left\{k_{n}^{(s)}\right\}$ also converge to some limits $v_{n}$ and $k_{n}$ respectively, and $\left\{w^{(s)}\left(r_{j}, t_{n}^{(s)}\right)\right\}$ converge to a limit function $w\left(r_{j}, t_{n}\right)$. (See (6.7).) Clearly $u\left(r_{j}, t_{n}\right) \equiv\left(1 / r_{j}\right) w\left(r_{j}, t_{n}\right) \quad\left(0 \leq j \leq J_{n}\right)$ and $k_{n}$ satisfy (5.1) at each $t_{n}$. Thus we have proved that our iteration procedure converges for some constant $\beta$.

\section{§ 7. Numerical Example}

We will show some numerical examples. We fix the pattern of the initial function (the initial temperature distribution) as

$$
f(r)=r\left(b^{2}-r^{2}\right) \quad(0<r<b)
$$

where we take $b=0.995$ and consider the four cases (i) $\gamma=1.0$, (ii) $\gamma=2.0$, (iii) $\gamma=5.0$ and (iv) $\gamma=10.0$. In any case we take $m=1.0$, 
$\alpha=1.0$ and $h=0.01$. For the iteration procedure we use the criterion of convergence

$$
\frac{k_{n}^{(s)}-k_{n}^{(s-1)}}{k_{n}^{(s)}} \mid<0.001
$$

and we take $\beta=0.001$ for the case (i), and $\beta=0.01$ for the case (ii), and $\beta=0.1$ for the cases (iii) and (iv). The iteration procedure with $\beta=0.01$ for the case (iii) resulted in divergence at $n=13$ in our computation.

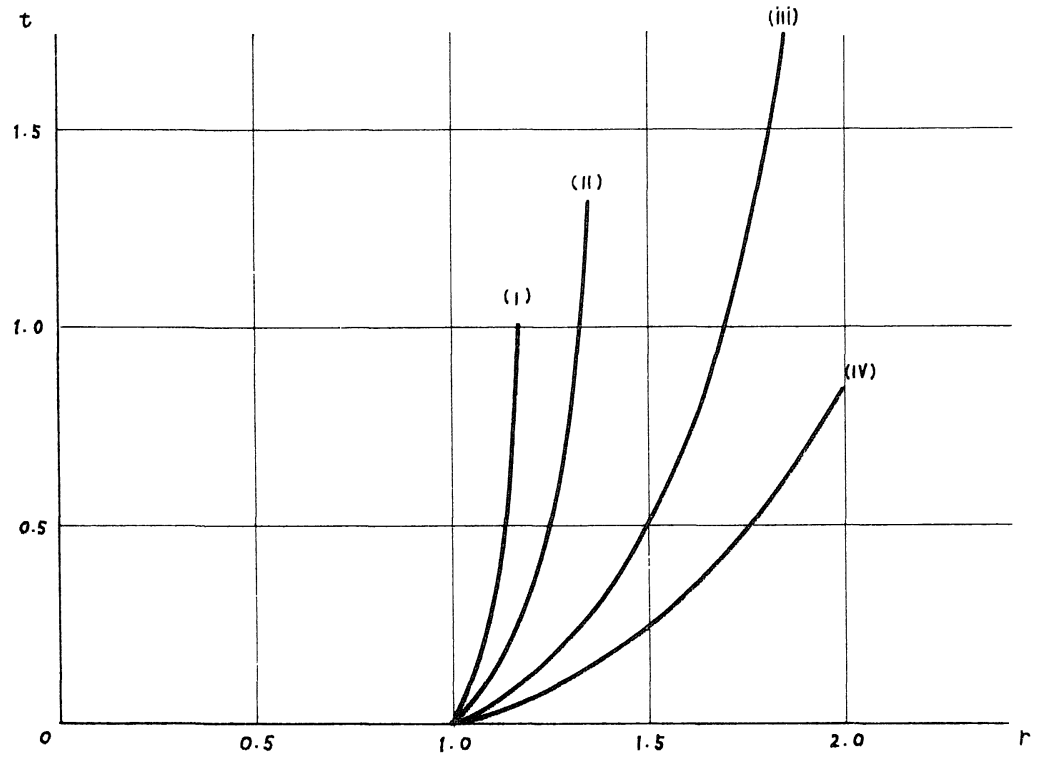

Fig. 1. Change of free boundaries for four cases

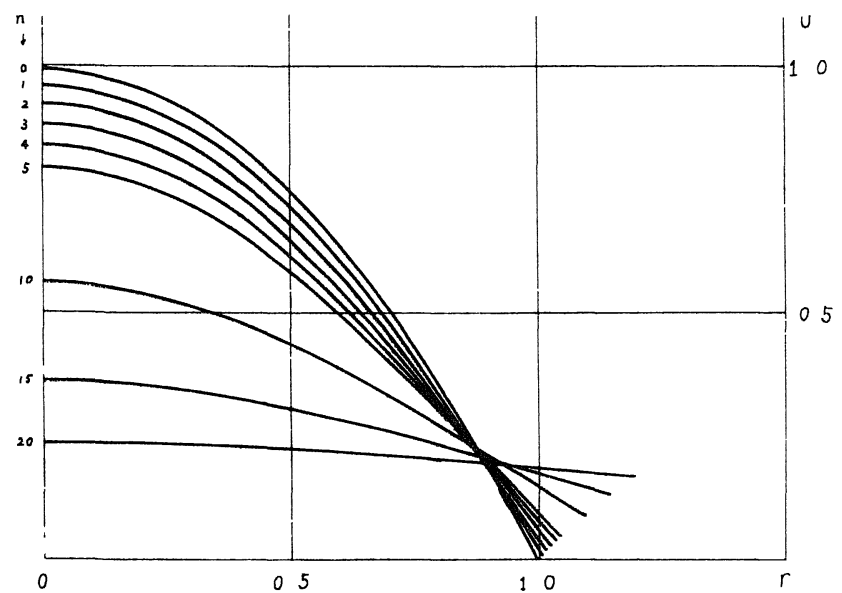

Fig. 2. Change of temperature distribution with time for the case (i) 


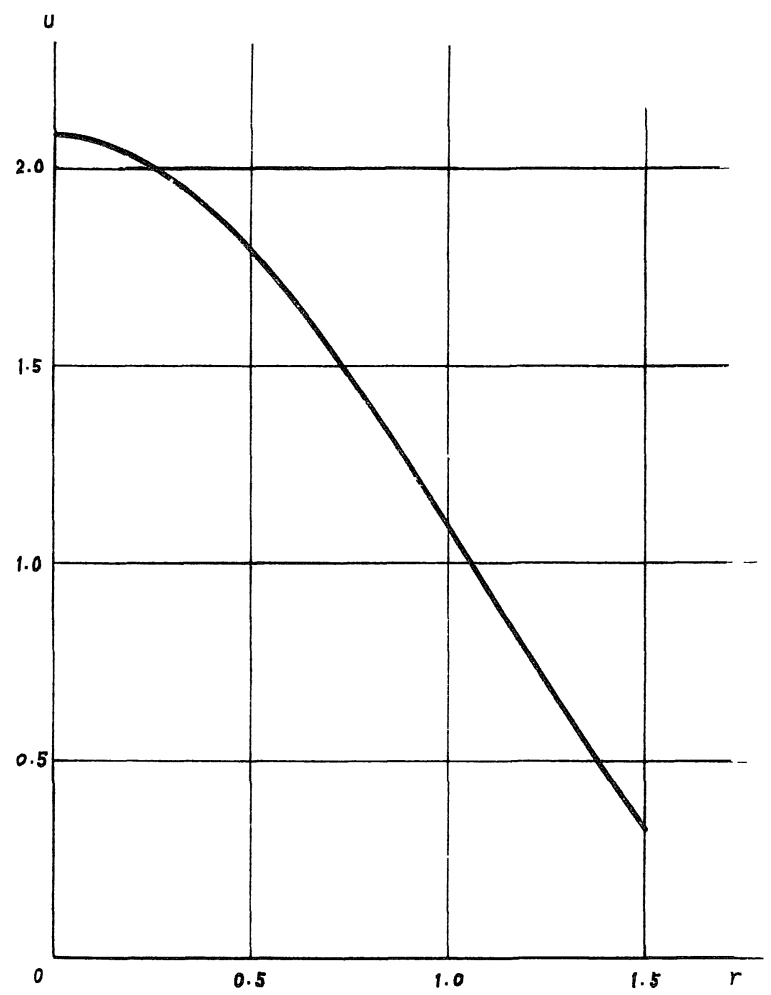

Fig. 3. Temperature distribution at $t=t_{50}=0.4964$ for the case (iii)

The Fig-1 shows the free boundaries for the four cases. The Fig-2 shows the change of temperature distribution with time $(n)$ for the case (i). The Fig-3 also shows the temperature distribution at $t=t_{50}=0.4963$ for the case (iii).

\section{Acknowledgement}

The author thanks Prof. M. Yamaguti for correcting some parts of his manuscript and constant encouragement, and Prof. N. Inoyama for instructing of undercooling metal and lending some references. And he is also indebted to Miss H. Shinoda for typewriting his manuscript.

\section{References}

[1] Flemings, M. C., Solidification processing, McGraw-Hill, Inc., 1974.

[2] Friedman, A., Free boundary problems for parabolic equations 1. Melting of solids, J. Math. and Mech., 8 (1959), 499-517. 
[3] Frideman, A., Free boundary problems for parabolic equations II. Condensation or evapolation of a liquid drop, J. Math. and Mech., 9 (1960), 19-66.

[4] Friedman, A.. Free boundary problems for parabolic equations III. Dissolution of a gas bubble in liquid, J. Math. and Mech., 9 (1960), 327-345.

[5] Cannon, J. R. and Hill, C. D., Existence, uniqueness, stability and monotone dependence in a Stefan problem for the heat equation, J. Math. and Mech., 17 (1967), $1-20$.

[6] Cannon, J. R., and Hill, C. D., Remarks on a Stefan problem, J. Math. and Mech., 17 (1967), 433-442.

[7] Cannon, J. R., Hill, C. D. and Primicerio, M., The one-phase Stefan problem for the heat equation with boundary temperature specification, Arch. Rat. Mech. Anal., 39 (1970), 270-274.

[8] Friedman, A., Remarks on the maximum principle for parabolic equations and its applications, Pacific J. of Math., 8 (1958), No. 2, 201-211.

[9] Nogi, T., A difference scheme for solving the Stefan problem, Publ. Res. Inst. Math. Sci., Kyoto Univ., 9 (1974), 543-575.

[10] Petrowsky, J. G., Partial Differential Equations, Originally published in Moscow in 1961 by Fizmatgiz under the title Lektsii ob urarneniyah s chastnumi proizvodnumi. 
